\title{
Brown fat activation mitigates alcohol-induced liver steatosis and injury in mice
}

\author{
Hong Shen, ${ }^{1}$ Lin Jiang, ${ }^{1}$ Jiandie D. Lin, ${ }^{2}$ M. Bishr Omary, ${ }^{1,3}$ and Liangyou Rui ${ }^{1,3}$ \\ 'Department of Molecular \& Integrative Physiology, ${ }^{2}$ Life Sciences Institute and Department of Cell \& Developmental Biology, and ${ }^{3}$ Department of Internal Medicine, Division of Gastroenterology and \\ Hepatology, University of Michigan Medical School, Ann Arbor, Michigan, USA.
}

\begin{abstract}
Chronic alcohol consumption causes liver injury, inflammation, and fibrosis, thereby increasing morbidity and mortality. Paradoxically, modest drinking is believed to confer metabolic improvement, but the underlying mechanism remains elusive. Here, we have identified a hepatoprotective brain/brown adipose tissue (BAT)/liver axis. Alcohol consumption or direct alcohol administration into the brain stimulated hypothalamic neural circuits and sympathetic nerves innervating BAT and dramatically increased BAT uncoupling protein 1 (Ucp1) expression and activity in a BAT-sympathetic nerve-dependent manner. BAT and beige fat oxidized fatty acids to fuel Ucp1-mediated thermogenesis, thereby inhibiting lipid trafficking into the liver. BAT also secreted several adipokines, including adiponectin, which suppressed hepatocyte injury and death. Genetic deletion of Ucp1 profoundly augmented alcohol-induced liver steatosis, injury, inflammation, and fibrosis in male and female mice. Conversely, activation of BAT and beige fat through cold exposure suppressed alcoholic liver disease development. Our results unravel an unrecognized brain alcohol-sensing/sympathetic nerve/BAT/liver axis that counteracts liver steatosis and injury.
\end{abstract}

\section{Introduction}

Alcohol abuse accounts for $3.8 \%$ of deaths and $4.6 \%$ of disability globally $(1,2)$. Chronic heavy alcohol drinking causes many adverse health consequences, including alcoholic liver disease (ALD). Several processes have been identified as being involved in ALD pathogenesis. Alcohol intake stimulates adipose lipolysis, thus increasing lipid trafficking into the liver to induce steatosis (3-8). Alcohol consumption also promotes hepatic de novo lipogenesis, further worsening liver steatosis (9). Alcohol and its metabolites directly cause hepatocyte damage, resulting in liver injury and inflammation (10). Alcohol causes dysbiosis and impairment in intestinal barriers, further increasing liver damage risk $(11,12)$. Counterintuitively, modest drinking is known to confer health benefits, including protections against cardiovascular disease, type 2 diabetes, and nonalcoholic fatty liver disease (NAFLD) (13-22).

Adipose tissue profoundly influences liver metabolism and homeostasis. Humans and rodents have 3 different types of fat, white adipose tissue (WAT), brown adipose tissue (BAT), and beige fat. WAT stores excess energy as triacylglycerol (TAG) and releases free fatty acids (FFAs) to support energy metabolism during fasting. In contrast, BAT and beige fat oxidize FFAs and glucose to produce heat, thus maintaining body temperature homeostasis (23-25). BAT/beige fat thermogenesis is mediated mainly by uncoupling protein 1 (Ucp1), which is expressed in a restricted manner in brown

Related Commentary: p. 2181

Conflict of interest: The authors have declared that no conflict of interest exists. Copyright: (c) 2019, American Society for Clinical Investigation.

Submitted: August 21, 2018; Accepted: March 14, 2019; Published: April 29, 2019.

Reference information: / Clin Invest. 2019;129(6):2305-2317.

https://doi.org/10.1172/JCI124376. adipocytes and beige adipocytes $(23,25)$. Ucp1 resides in the inner mitochondrial membrane and mediates proton leaking across the membrane, thereby dissipating chemical energy as heat (24). Cold exposure potently activates, through the sympathetic nervous system (SNS), BAT/beige fat thermogenesis to combat heat loss in both rodents and humans (26-33). Importantly, BAT and beige fat also counteract body weight gain, obesity, and metabolic disease through increasing energy expenditure $(24,25,34,35)$. However, the role of BAT/beige fat in ALD is poorly understood.

In light of their ability to burn FFAs, we postulated that BAT and beige fat might serve as a sink for plasma FFAs, consequently suppressing liver steatosis. In this study, we used Ucp1-knockout $\left(U c p 1^{-/-}\right)$mice as a BAT/beige fat-defective animal model to test this hypothesis and used BAT/hepatocyte cocultures and BAT-conditioned media (CM-BAT) systems to identify BATderived endocrine factors responsible for hepatoprotection. Furthermore, we exploited direct alcohol administration into the brain and BAT denervation approaches to elucidate pathways that mediate alcohol activation of BAT. These comprehensive analyses unravel a previously unrecognized brain alcohol-sensing/ sympathetic nerve/BAT/liver axis that counteracts ALD.

\section{Results}

Ucp1 deficiency exacerbates ALD in male mice. To determine whether Ucp1-mediated thermogenesis ameliorates ALD, we placed $U c p 1^{-/-}$and $U c p 1^{+/+}$males on a Lieber-DeCarli diet supplemented with alcohol (5\%) for 7 to 8 weeks. Body weight and expression of Ucp2 or Ucp3 in BAT and WAT were normal in $U c p 1^{-/-}$mice (Supplemental Figure 1, A and B). Levels of plasma alanine aminotransferase (ALT) (liver injury marker) were significantly higher in $U c p 1^{-/-}$relative to $U c p 1^{+/+}$mice at 3 weeks after alcohol feeding (Figure 1A). Liver weight (Supplemental Figure 1C) and TAG levels 
(Figure 1B) were significantly higher, while hepatocyte lipid droplets were dramatically larger and more abundant (Figure 1C), in alcohol-fed $U c p 1^{-/-}$mice. Liver cell death was substantially higher in $U c p 1^{-/-}$than in $U c p 1^{+/+}$mice, as assessed by TUNEL assays and immunostaining of liver sections with antibodies against cleaved caspase 3 (apoptosis marker) and Rip3 (necrosis marker) (Figure 1, $\mathrm{C}$ and D). The levels of cleaved caspase- 3 and Rip3 in liver extracts were substantially higher in $U c p 1^{1 /-}$ mice (Figure $1 \mathrm{E}$ ).

Liver injury is known to trigger compensatory hepatocyte proliferation (36); accordingly, the number of liver Ki67 $7^{+}$proliferating cells was significantly higher in $U c p 1^{-/-}$relative to $U c p 1^{+/+}$mice (Figure $1, \mathrm{C}$ and D). Liver inflammation was more severe in $U c p 1^{-/-}$than in $U c p 1^{+/+}$mice, as assessed by hepatic myeloperoxidase-expressing (MPO-expressing) neutrophil number and cytokine expression (Figure 1, C and D, and Supplemental Figure 1D). Liver oxidative stress, as assessed by nitrotyrosine (Figure 1, C and D) and ROS levels (Figure $1 \mathrm{~F}$ ), was markedly higher in $U c p 1^{-/-}$relative to $U c p 1^{+/+}$mice. Liver fibrosis (Sirius red staining) was undetectable in alcohol-fed $U c p 1^{+/+}$mice; strikingly, we detected abundant liver fibrosis in $U c \mathrm{c}^{-/-}$mice (Figure 1, C and D). Levels of liver hydroxyproline (fibrosis marker) and expression of fibrosis-related genes (e.g., collagen 1a1 and Timp1) were significantly higher in $U c p 1^{-1-}$ than in $U c p 1^{+/+}$mice (Figure $1 \mathrm{G}$ and Supplemental Figure 1D). To extend these findings, we analyzed $\alpha$-smooth muscle actinexpressing ( $\alpha$-SMA-expressing) myofibroblasts responsible for fibrosis $(17,37)$. Liver $\alpha-\mathrm{SMA}^{+}$myofibroblast numbers and $\alpha$-SMA expression were substantially higher in $U c p 1^{-/-}$relative to $U c p 1^{+/+}$ mice (Figure 1, C-E). Aberrant cholangiocyte proliferation (ductular reactions) is linked to morbidity and mortality (38). Cytokeratin 19-expressing (CK19-expressing) cholangiocyte numbers were higher in $U c p 1^{-/-}$mice (Figure 1, C and D). Notably, body weight, liver lipid levels, and liver integrity were normal in $U c p 1^{-/-}$mice placed on the Lieber-DeCarli diet supplemented with maltose (Supplemental Figure 1, E-G). To further confirm the hepatoprotective function of Ucp1, we placed $U c p 1^{-/-}$mice on an alcohol diet superimposed with multiple bouts of binge drinking (39). Body weight was comparable between $U c p 1^{-/-}$and $U c p 1^{1^{+/}}$mice (Supplemental Figure 2A). Plasma ALT levels became markedly higher in $U c p 1^{-/-}$mice at 20 days after alcohol feeding (Supplemental Figure 2B). Liver steatosis, oxidative stress, injury, inflammation, and fibrosis were substantially more severe in $U c p 1^{-/-}$relative to $U c p 1^{+/+}$ mice (Supplemental Figure 2, C and D). Collectively, these results demonstrate that BAT and beige fat protect against ALD in males.

Ucp1 deficiency worsens ALD in female mice. To determine whether male and female sex influence the ability of BAT to protect against ALD, we placed $U c p 1^{+/+}$and $U c p 1^{-/-}$female mice on an alcohol diet for 7 to 8 weeks. Body weight was comparable between these 2 groups (Supplemental Figure 3A). $U c p 1^{-/-}$females, like $U c p 1^{-1-}$ males, developed more severe ALD than $U c p 1^{+/+}$females. Plasma ALT levels became significantly higher in $U c p 1^{-/-}$relative to $U c p 1^{+/+}$ females at 30 days after alcohol feeding (Figure 2A). Liver TAG levels were higher, while hepatocyte lipid droplets were larger and more abundant in $U c p 1^{-/-}$females (Figure 2, B-D). Liver oxidative stress (e.g., ROS and nitrotyrosine levels), hepatocyte death (e.g., $\mathrm{TUNEL}^{+}$, caspase $3^{+}$, and Rip3 ${ }^{+}$cells), compensatory hepatocyte proliferation (Ki67 ${ }^{+}$cells), ductular reactions (e.g., higher $\mathrm{CK} 19^{+}$ cholangiocyte number), liver inflammation (e.g., higher $\mathrm{MPO}^{+}$ neutrophil number), and liver fibrosis (e.g., increases in Sirius red areas, hydroxyproline content, and $\alpha$-SMA ${ }^{+}$myofibroblast number) were markedly higher in $U c p 1^{-/-}$than in $U c p 1^{+/+}$females (Figure 2, C-D). The levels of liver cleaved caspase-3, Rip3, and $\alpha$-SMA protein were substantially higher in alcohol-fed $U c p 1^{-/-}$relative to $U c p 1^{+/+}$females (Figure 2E). Liver ROS and hydroxyproline levels were also significantly higher in $U c p 1^{-/-}$females (Figure 2, $\mathrm{F}$ and G). To further confirm protection against ALD by Ucp1, we placed $U c p 1^{-/-}$female mice on an alcohol diet for 6 weeks superimposed with multiple bouts of binge drinking. Plasma ALT levels, liver steatosis, hepatic oxidative stress, liver cell death and compensatory proliferation, ductular reactions, hepatic inflammation, and liver fibrosis were markedly higher in $U c p 1^{1 /-}$ relative to $U c p 1^{+/+}$females (Supplemental Figure 3, B-F). These results indicate that BAT and beige fat also protect female mice from ALD.

Ucp1 deficiency augments acute binge alcohol-induced hypothermia and liver damage. To determine whether Ucp1 also confers protection against acute alcohol-induced liver injury, we administrated a single bout of binge alcohol into $U c p 1^{-/-}$and $U c p 1^{+/+}$mice. Surprisingly, all of the $U c p 1^{-/-}$mice died within 36 hours following binge drinking, whereas all of the $U c p 1^{+/+}$mice survived (Figure $3 \mathrm{~A}) . U c p 1^{-/-}$but not $U c p 1^{+/+}$mice developed life-threatening hypothermia after binge alcohol intake (Figure 3B). Body surface temperatures were also lower in $U c p 1^{-/-}$than in $U c p 1^{+/+}$mice (Supplemental Figure 4A). We harvested livers at 24 to 36 hours after binge drinking. Plasma ALT levels, liver steatosis (liver TAG levels and lipid droplet number and size), liver oxidative stress (nitrotyrosine and ROS levels), liver cell death (TUNEL ${ }^{+}$, Rip3 ${ }^{+}$, caspase $3^{+}$cells), compensatory hepatocyte proliferation (Ki67 ${ }^{+}$cells), and liver inflammation $\left(\mathrm{MPO}^{+}\right.$neutrophil number and cytokine expression) were substantially higher in $U c p 1^{-/-}$relative to $U c p 1^{+/+}$ mice (Figure 3, C-H). Alcohol intoxication is known to cause fatal hypothermia in cold environments in humans (40), prompting us to determine whether hypothermia causes alcohol-induced death of $U c p 1^{-/-}$mice. Alcohol increases heat loss by stimulating cutaneous vasodilation and skin blood circulation (40), so we raised the ambient temperature from $22^{\circ} \mathrm{C}$ to $30^{\circ} \mathrm{C}$ to reduce heat loss. Indeed, core temperature was indistinguishable between alcoholfed $U c p 1^{-/-}$and $U c p 1^{+/+}$mice at thermoneutrality (Supplemental Figure 4B). Remarkably, all Ucp1 $1^{-/}$mice survived following binge alcohol intake $(n=6)$. Likewise, acute binge alcohol intake also induced more severe liver steatosis and oxidative stress in $\mathrm{Ucp1}^{-/-}$ mice at thermoneutrality $\left(30^{\circ} \mathrm{C}\right)$ (Supplemental Figure $\left.4 \mathrm{C}\right)$. Collectively, these results indicate that BAT and beige fat crucially protect against ALD in a body weight-independent manner.

BAT suppresses lipid trafficking into the liver and secretes hepatoprotective adipokines. Given that alcohol intake stimulates adipose lipolysis $(3,4,6,41)$, we determined whether BAT impedes trafficking of circulating FFAs into the liver. Chronic alcohol intake increased phosphorylation and activation of WAT hormonesensitive lipase (HSL) in both $U c p 1^{-/-}$and $U c p 1^{+/+}$mice (Supplemental Figure 5A), suggesting that WAT lipolysis is relatively normal in $U c p 1^{-/-}$mice. Plasma FFA levels were comparable between $U c p 1^{-/-}$and $U c p 1^{+/+}$mice under either baseline or alcohol-treated conditions (Supplemental Figure 5B), suggesting that the liver rapidly absorbs FFAs and consequently masks plasma FFA elevations. In line with this notion, liver expression of CD36, which mediates 

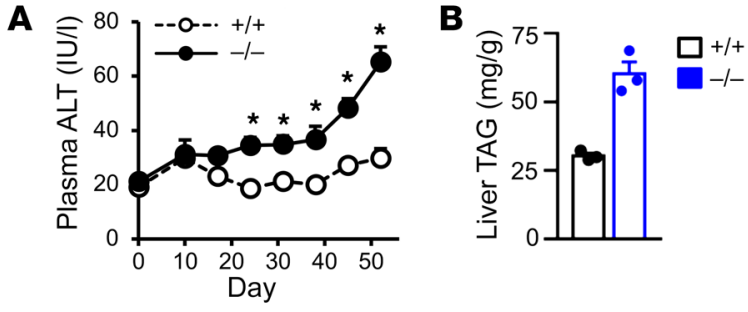

D
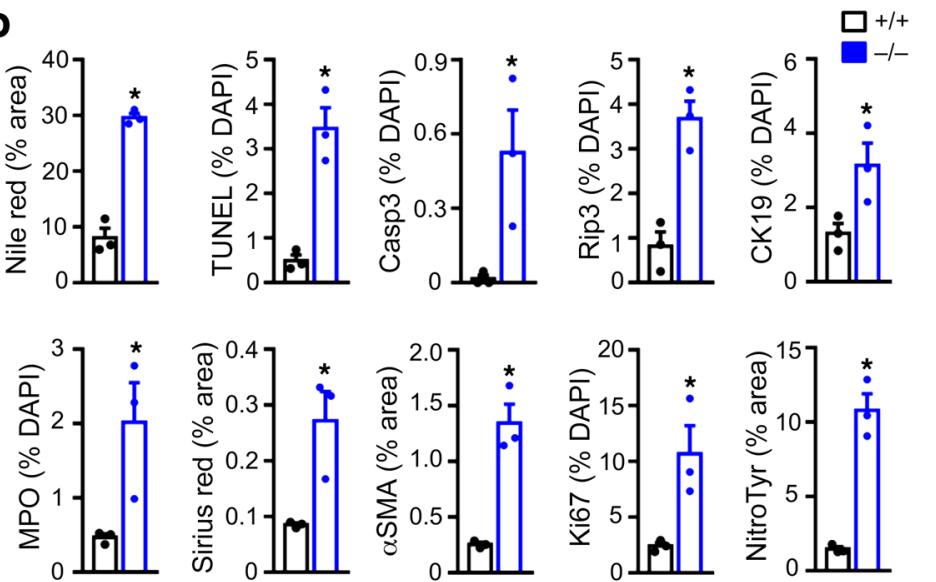

E

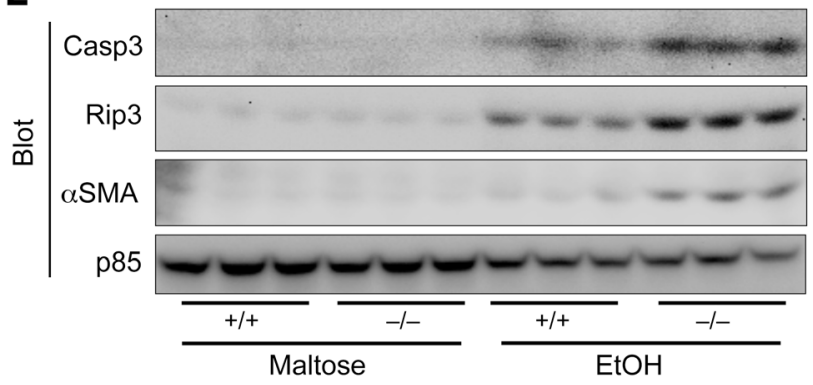

$\mathbf{F}$

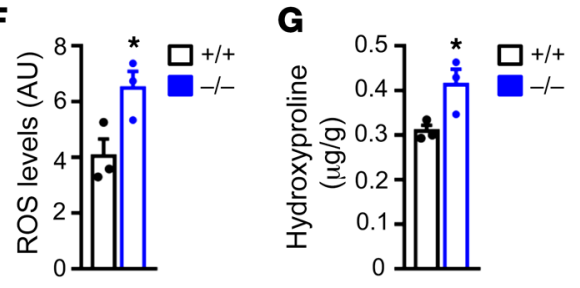

Figure 1. Ucp1 deficiency exacerbates ALD in males. Ucp $1^{+/+}$and $U c p 1^{-1-}$ males were fed an alcohol (EtOH) diet for 7 to 8 weeks (housed at $22^{\circ} \mathrm{C}$ ).

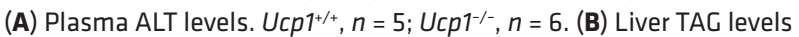
(normalized to liver weight, $n=3$ mice per group). (C, D) Liver sections were stained with the indicated reagents. Positive signals were quantified ( $n=3$ mice per group). Scale bars: $200 \mu \mathrm{m}$. (E) Liver extracts were immunoblotted with the indicated antibodies. (F, G) Liver ROS and hydroxyproline levels (normalized to liver weight, $n=3$ mice per group). Data are presented as mean \pm SEM. ${ }^{*} P<0.05$, 2-tailed unpaired Student's $t$ test.
C

崖
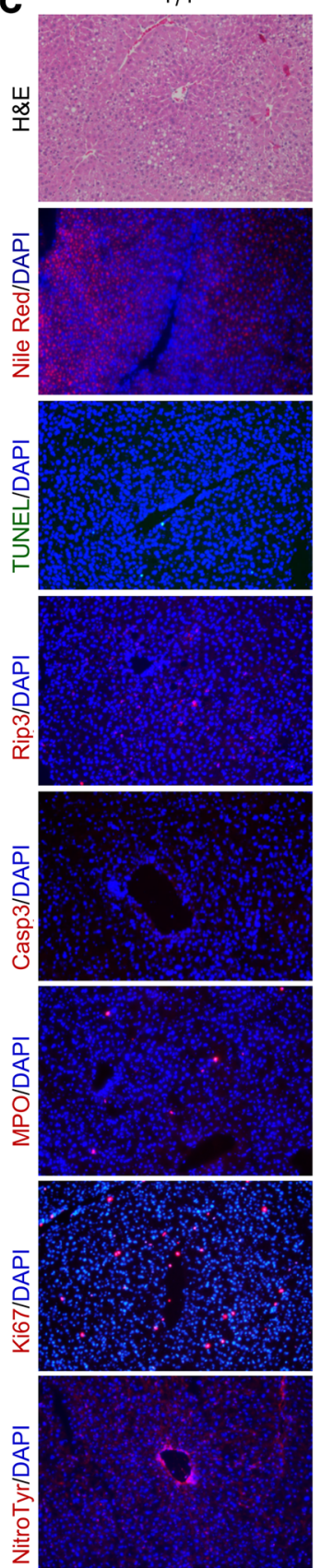

덩
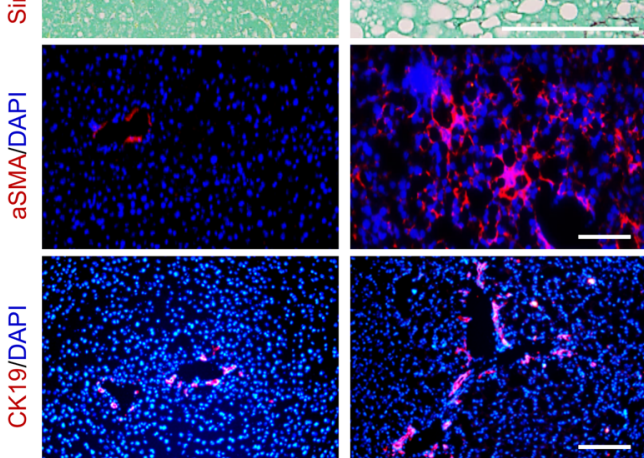
A

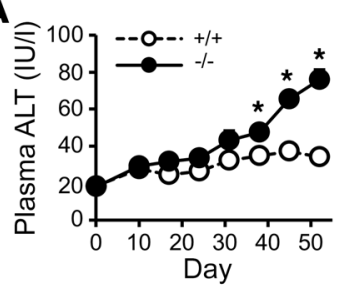

B

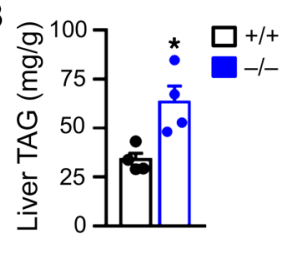

D
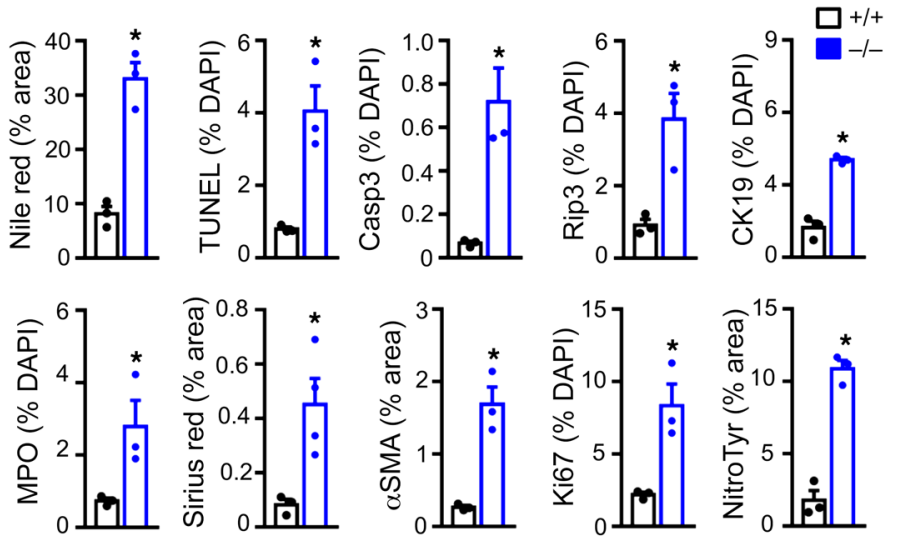

$\mathbf{E}$

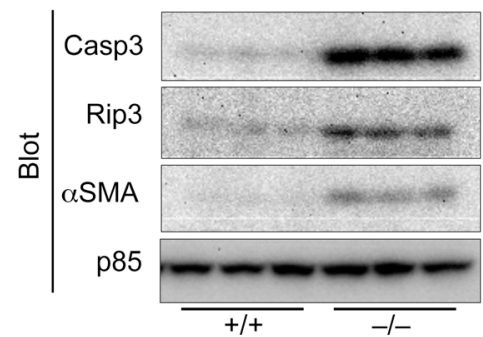

$\mathbf{F}$

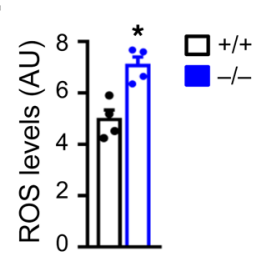

G

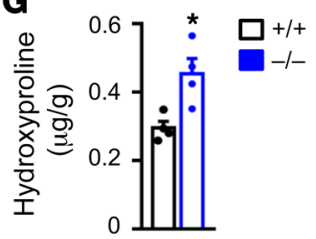

C
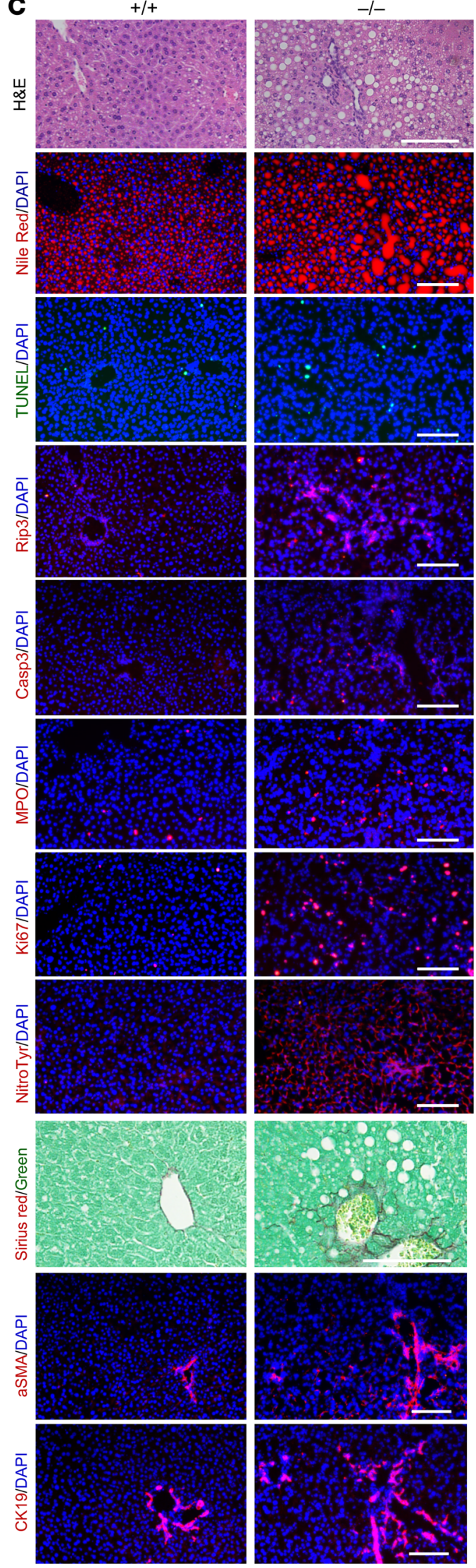

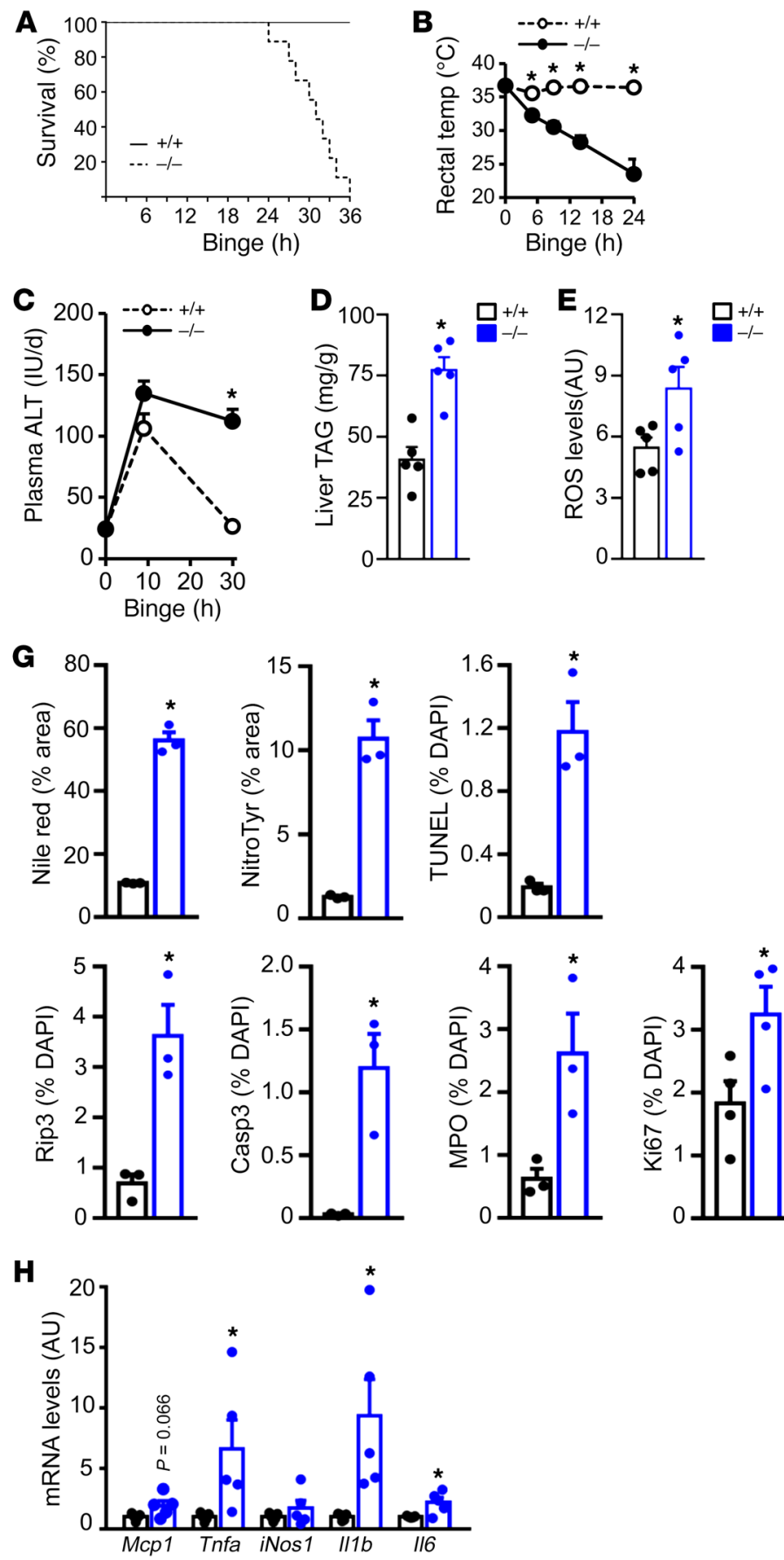
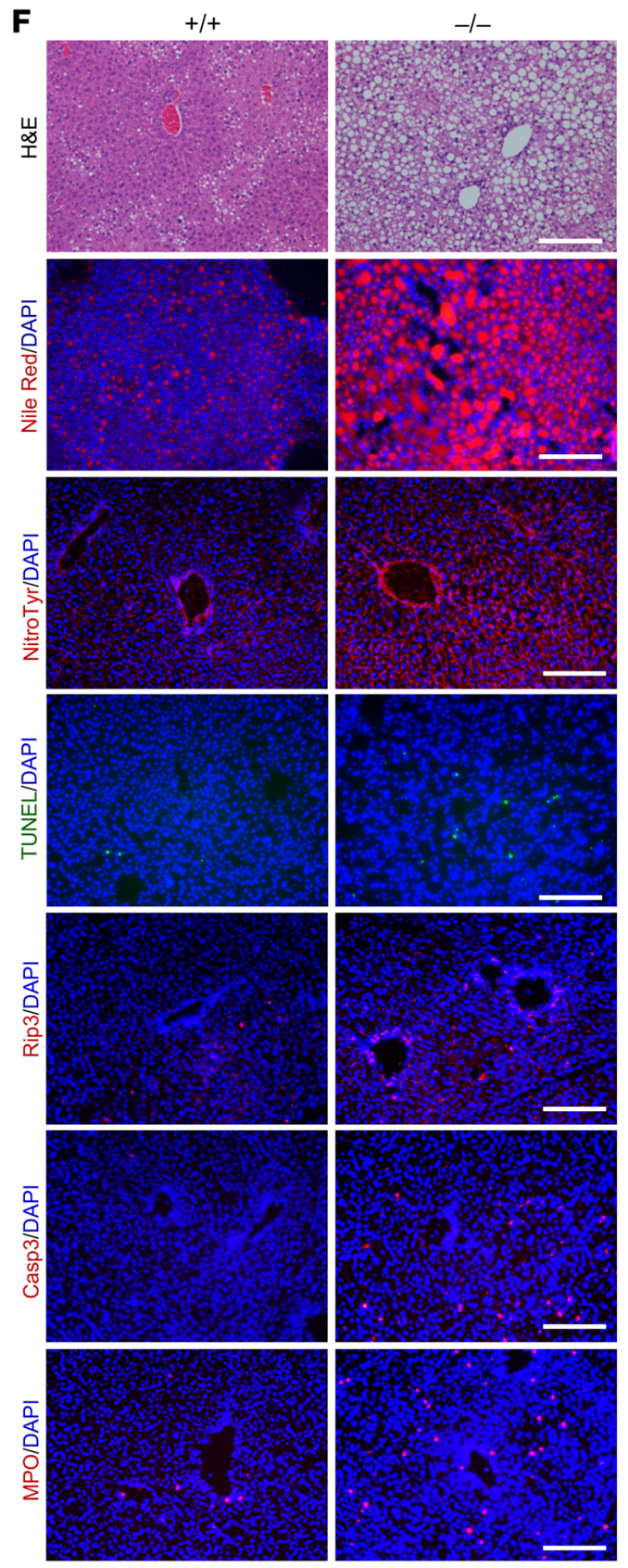

Figure 3. Ucp 1/- mice develop fatal hypothermia and hepatitis in response to binge drinking. Male mice were treated with binge alcohol, and livers were harvested 24 to 36 hours later. (A) Survival rates ( $n=9$ per group). (B) Rectal temperature ( $n=4$ per group). (C) Plasma ALT activity ( $n=5$ per group). (D) Liver TAG levels (normalized to liver weight, $n=5$ mice per group). (E) Liver ROS levels (normalized to liver weight, $n=5$ mice per group). (F, G) Liver sections were stained with the indicated reagents. Positive signals were quantified ( $n=3-4$ mice per group). Scale bars: $200 \mu \mathrm{m}$. (H) Liver gene expression (normalized $18 \mathrm{~S}$ levels, $n=5$ mice per group). Data are presented as mean \pm SEM. ${ }^{*} P<0.05$, 2-tailed unpaired Student's $t$ test.

FFA uptake, was significantly higher in $U c p 1^{-/-}$relative to $U c p 1^{+/+}$ mice following chronic alcohol consumption (Supplemental Figure 1D). To further confirm BAT inhibition of lipid trafficking into the liver, $U c p 1^{1 /-}$ mice were treated with $\beta_{3}$ adrenergic agonist CL316243, which stimulates both WAT lipolysis and BAT thermogenesis. Liver steatosis was substantially more severe in $U c p 1^{1-}$ relative to $U c p^{+/-}$mice (Supplemental Figure $5 \mathrm{C}$ ). Therefore, BAT alleviates liver steatosis at least in part by burning plasma FFAs, thereby decreasing lipid trafficking into the liver.

We reasoned that BAT might also protect against liver injury via adipokines. Primary hepatocytes were cocultured with BAT or WAT explants, and hepatocyte death was induced by palmitic acid (PA) treatment. Remarkably, BAT, but not WAT, blocked PA-induced hepatocyte apoptosis (Figure 4A). Similarly, CM-BAT 


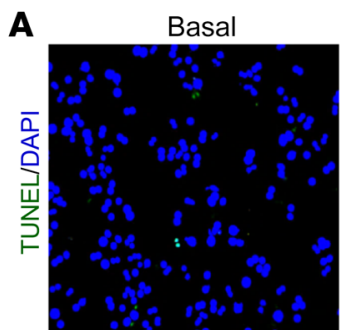

B

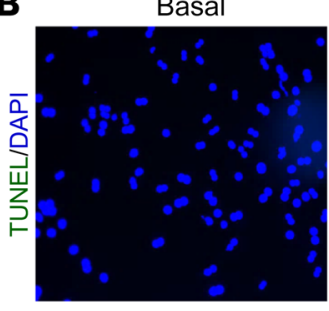

C

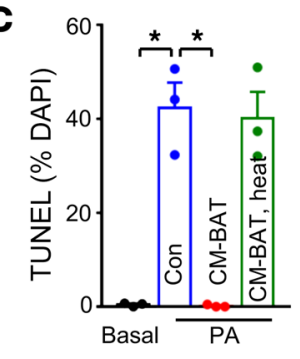

F
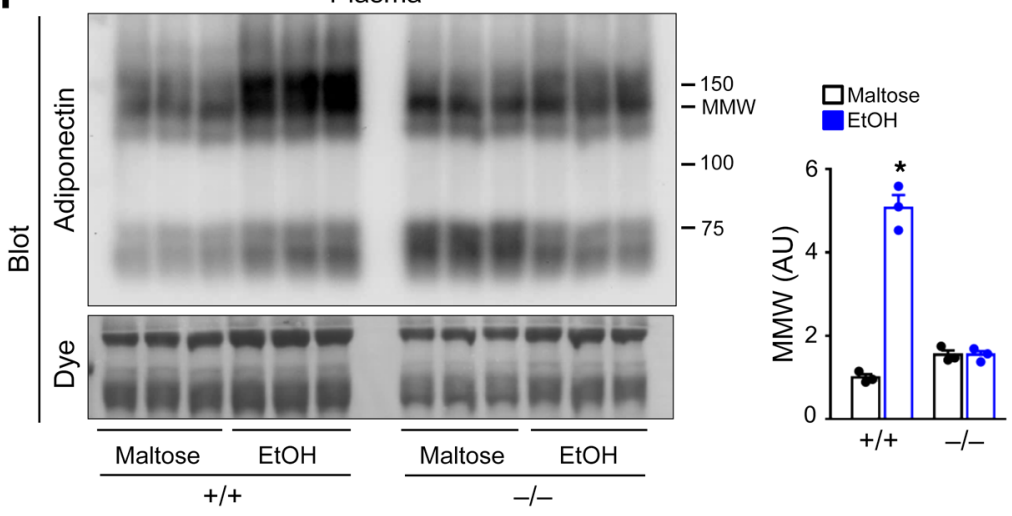

H

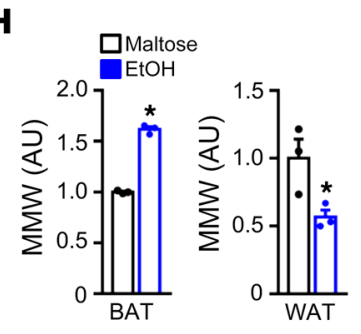

PA, Con

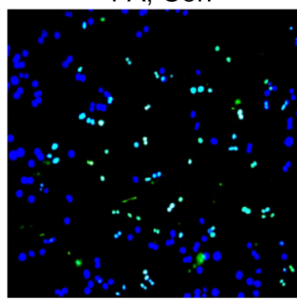

PA, Con

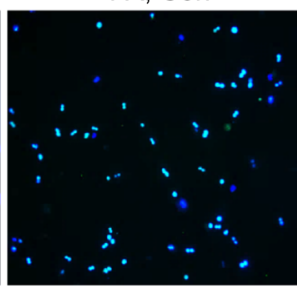

D
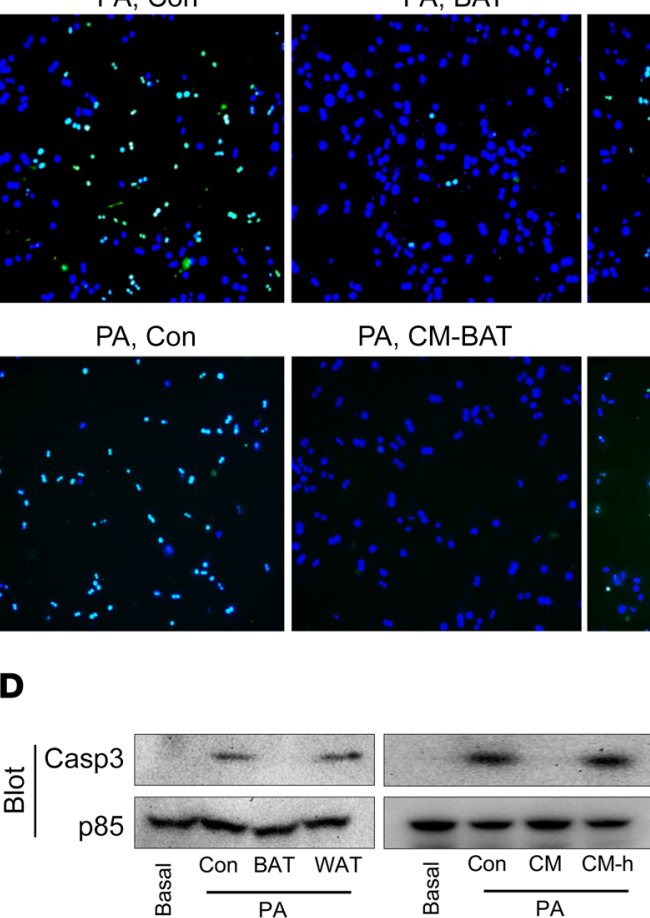

PA, CM-BAT
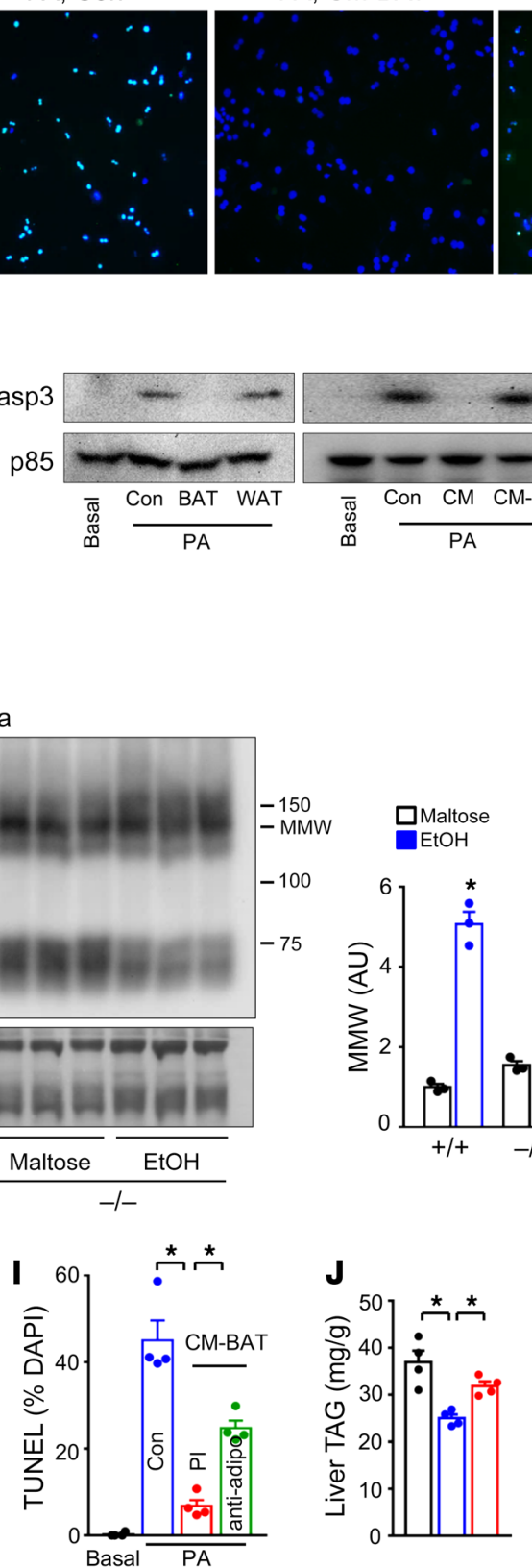

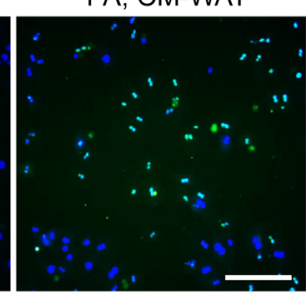

$\mathbf{E}$

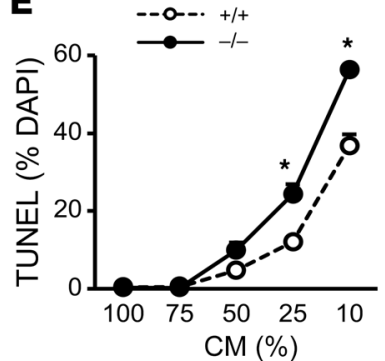

G

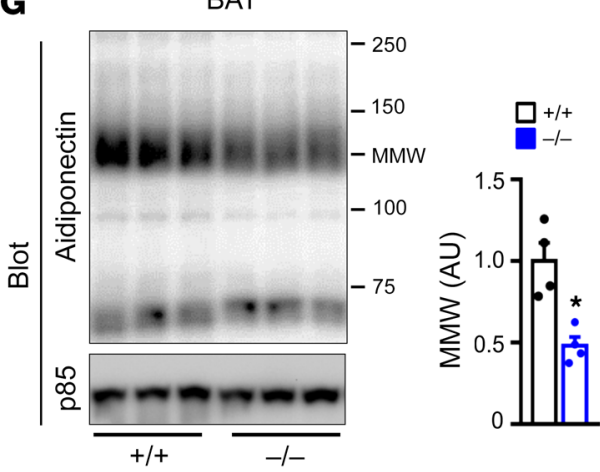

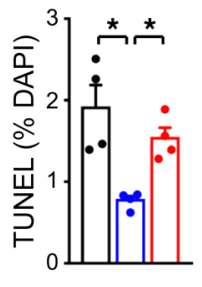

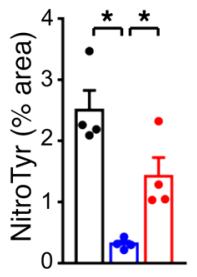

$\square 30^{\circ} \mathrm{C}$
$-8^{\circ} \mathrm{C}, \mathrm{P}$

$8^{\circ} \mathrm{C}$, anti-adiponectin 
Figure 4. BAT secretes hepatoprotective adipokines. (A-C) Primary hepatocytes were cocultured for 24 hours without (Con) or with BAT or WAT explants (A), BAT (CM-BAT) or WAT (CM-WAT) conditioned medium (B), or heat-inactivated conditioned medium (C) in the presence of PA and then subjected to TUNEL assays ( $n=3$ mice per group). Scale bars: 200 $\mu \mathrm{m}$. (D) Primary hepatocytes were treated without (Con) or with CM-BAT or CM-WAT for 24 hours. Cell extracts were immunoblotted with the indicated antibodies. (E) CM-BAT was prepared from $\operatorname{Ucp}^{+/+}(n=3)$ and $U c p 1^{1--}(n=$ 3) males (on alcohol diet for 10 days). Primary hepatocytes were treated with PA and CM-BAT. TUNEL assays were performed 24 hours later. (F, G) $U c p 1^{1 /+}$ and $U c \mathrm{cp}^{-/-}$mice were fed an alcohol or maltose diet for 7 weeks.

(F) Plasma protein was stained with anti-adiponectin antibody or ponceau $S$ dye. MMW levels were normalized to ponceau S levels. (C) BAT extracts were immunoblotted with antibodies against adiponectin or p85. MMW levels were normalized to p85 levels. (H) C57BL/6 males were fed an alcohol or maltose diet for 7 weeks. MMW levels were assessed in BAT and epididymal WAT by immunoblotting (normalized to p85 levels, $n=3$ per group). (I) CM-BAT was pretreated with anti-adiponectin antibody or PI. Primary hepatocytes were treated without (Con) or with CM-BAT in the presence of PA. TUNEL assays were performed 24 hours later $(n=4$ mice per group). (J) $\mathrm{C} 57 \mathrm{BL} / 6$ males were exposed to cold $\left(8^{\circ} \mathrm{C}\right)$, binge alcohol, and anti-adiponectin antibody (PI as control). Liver TAG (normalized to liver weight), cell death (TUNEL), and oxidative stress (nitrotyrosine areas) were assessed ( $n=4$ per group). Data are presented as mean \pm SEM. Difference was analyzed by 2-tailed unpaired Student's $t$ test (between 2 groups) or 1-way ANOVA (between multiple groups). ${ }^{*} P<0.05$.

but not WAT (CM-WAT) fully suppressed PA-elicited hepatocyte apoptosis (Figure 4B). Consistently, BAT explants and CM-BAT, but not WAT explants and CM-WAT, abrogated the ability of PA to activate hepatocyte caspase-3 (Figure 4D). Heat pretreatment abolished the ability of CM-BAT to suppress hepatocyte death and caspase-3 activation (Figure 4, C and D), indicating that BAT secretes heat-labile hepatoprotective factors. Importantly, Ucp1 deficiency impaired the ability of BAT to protect against PAinduced hepatocyte death; nonetheless, $U c p 1^{-/-} \mathrm{CM}-\mathrm{BAT}$ at high concentrations ( $>75 \%$ ) still blocked hepatocyte death (Figure $4 \mathrm{E}$ ).

We postulated that adiponectin, which is known to protect against ALD (42), might mediate BAT protection against hepatocyte death. Chronic alcohol consumption increased plasma adiponectin levels, particularly medium molecular weight (MMW) adiponectin levels, in $U c p 1^{1^{++}}$but not $U c p 1^{-/-}$mice (Figure $4 \mathrm{~F}$ ). BAT MMW expression was significantly lower in alcohol-fed $U c p 1^{-/-}$relative to $U c p 1^{+/+}$mice (Figure $4 \mathrm{G}$ ). Unexpectedly, alcohol intake increased BAT MMW levels while decreasing WAT MMW levels (Figure 4H and Supplemental Figure 6A). Ucp1 deficiency increased BAT oxidative stress (Supplemental Figure 6B), prompting us to determine whether ROS causes a reduction in BAT MMW. We treated in vitro differentiated brown adipocytes with antioxidant $\mathrm{N}$-acetylcysteine (NAC) or with $\mathrm{H}_{2} \mathrm{O}_{2}$. NAC considerably increased, whereas $\mathrm{H}_{2} \mathrm{O}_{2}$ dramatically decreased, MMW levels in $U_{c p 1^{-/}}$brown adipocytes (Supplemental Figure 6, C and D). Thus, Ucp1 promotes adiponectin MMW production at least in part through reducing BAT oxidative stress. To determine whether adiponectin mediates hepatoprotection, we pretreated CM-BAT with anti-adiponectin antibody to deplete this adipokine. Preimmune serum (PI) was used as control. Depletion of adiponectin significantly reduced the ability of CM-BAT to suppress PAinduced hepatocyte death (Figure 4I and Supplemental Figure $6 \mathrm{E})$. To verify that adiponectin mediates BAT protection again
ALD in vivo, we treated C57BL/6 mice with anti-adiponectin antibody while placing them in a cold environment in order to activate BAT. Remarkably, cold activation of BAT substantially attenuated alcohol-induced liver steatosis, hepatic oxidative stress, and liver cell death; adiponectin neutralization significantly attenuated cold protection against ALD (Figure 4J and Supplemental Figure 6, $\mathrm{F}-\mathrm{H})$. In addition to adiponectin, IL-6 was also involved in protection against hepatocyte death by brown adipocytes (Supplemental Figure 7, A and B). Collectively, these results indicate that BATderived adipokines (e.g., adiponectin and IL-6) ameliorate ALD.

Alcohol consumption robustly activates BAT. We next sought to determine whether and how alcohol intake activates BAT. Chronic alcohol consumption considerably increased BAT Ucp1 levels in C57BL/6 mice housed at standard room temperature (Supplemental Figure 8A). Raising the ambient temperature is known to cause BAT inactivation and whitening (43). We confirmed that at thermoneutrality $\left(30^{\circ} \mathrm{C}\right)$, BAT became inactive, with enlarged lipid droplets and decreased Ucp1 expression (Figure 5, A and B). Remarkably, chronic alcohol consumption completely prevented warmth-induced whitening and inactivation of BAT (Figure 5A and Supplemental Figure 8B). BAT levels of Ucp1 and mitochondrial respiration complexes I, II, III, IV, and V were substantially higher in alcohol-fed mice housed at $30^{\circ} \mathrm{C}$ relative to maltose-fed mice housed at either $30^{\circ} \mathrm{C}$ or $22^{\circ} \mathrm{C}$ (Figure $5 \mathrm{~B}$ ). Alcohol feeding also increased BAT Ucp1 mRNA levels (Supplemental Figure 8C). Accordingly, alcohol consumption markedly increased BAT activity, as assessed by $\mathrm{O}_{2}$ consumption rates (OCR). OCR was significantly lower in maltose-fed mice at $30^{\circ} \mathrm{C}$ relative to $22^{\circ} \mathrm{C}$; alcohol consumption significantly increased OCR (Figure 5C). Notably, alcohol consumption also promoted beige fat induction at thermoneutrality (i.e., Ucp1 expression) (Figure 5D). To further confirm alcohol activation of BAT in vivo, we performed cold tolerance tests on mice housed at $22^{\circ} \mathrm{C}$. Body core temperatures were considerably higher in alcohol-fed relative to maltose-fed mice following cold exposure (Figure 5E). Energy expenditure, as estimated by $\mathrm{O}_{2}$ consumption and $\mathrm{CO}_{2}$ production, was also higher in alcohol-fed relative to maltose-fed mice, while respiratory exchange rates (RER) were lower in the alcohol group (Figure 5F). Alcohol consumption decreased body weight at thermoneutrality (Supplemental Figure 8D), presumably by increasing BAT/beige fat energy expenditure. Therefore, chronic alcohol intake potently activates BAT and beige fat, particularly in warm environments.

Alcohol activates a brain/sympathetic nerve/BAT/liver axis. We next set out to identify alcohol target tissues. Primary brown adipocytes and in vitro differentiated brown adipocytes were stimulated with ethanol for 5 to 18 hours. Alcohol did not increase Ucp1 expression (Figure 6, A and B). Considering that alcohol modulates neuronal activity, we centrally administrated a low dose of alcohol ( $5 \mu \mathrm{l}$ of $40 \%$ ethanol, intracerebroventricularly) daily for 3 weeks. Mice were housed at thermoneutrality to reduce baseline BAT activity. Central alcohol injection, like alcohol feeding, largely blocked warm-induced whitening of BAT. BAT TAG levels were significantly lower (Figure 6C), while Ucp1 expression was substantially higher (Figure 6, D and E) in alcohol- relative to PBS-treated mice. Expression of multiple BAT markers (e.g., Pgc1 $\alpha$, Cidea, Dio2, Elolv3, and Prdm16) (Figure 6F) and BAT OCR (Figure 6G) were significantly higher in alcohol-treated mice. 
A Maltose $22^{\circ} \mathrm{C}$
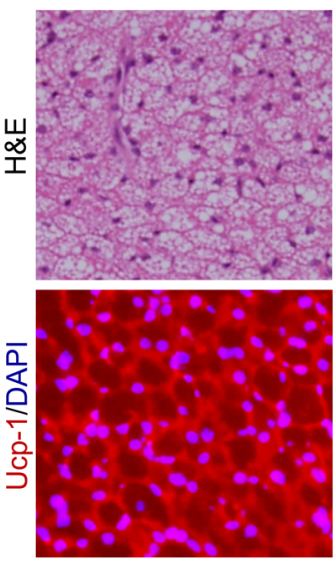

C
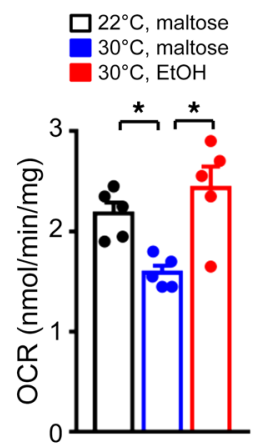

Maltose $30^{\circ} \mathrm{C}$
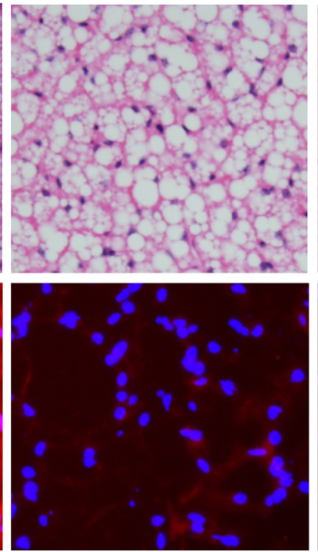

D

$22^{\circ} \mathrm{C}$, maltose
$30^{\circ} \mathrm{C}$, maltose $30^{\circ} \mathrm{C}, \mathrm{EtOH}$

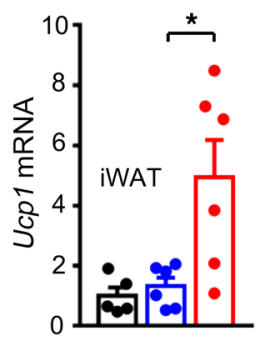

$\mathrm{EtOH}, 30^{\circ} \mathrm{C}$

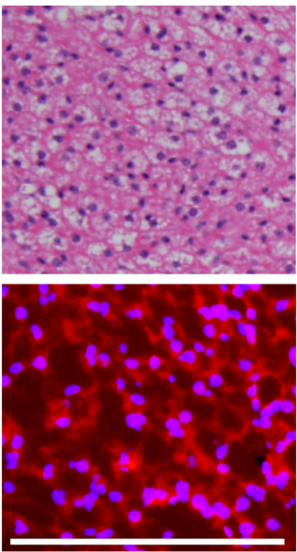

B

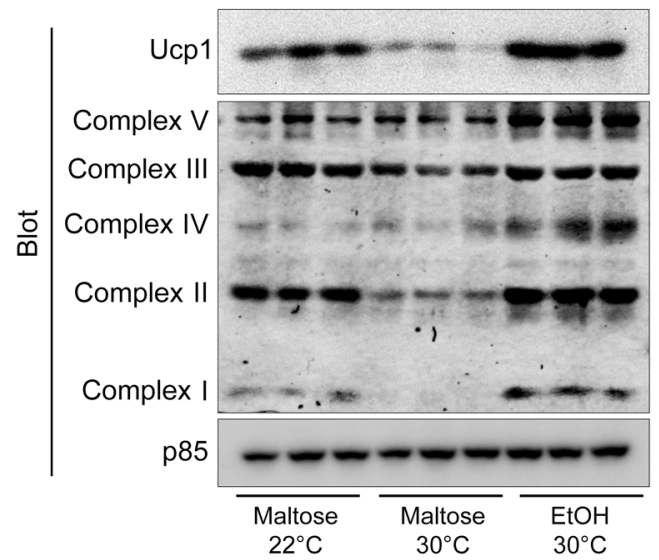

E

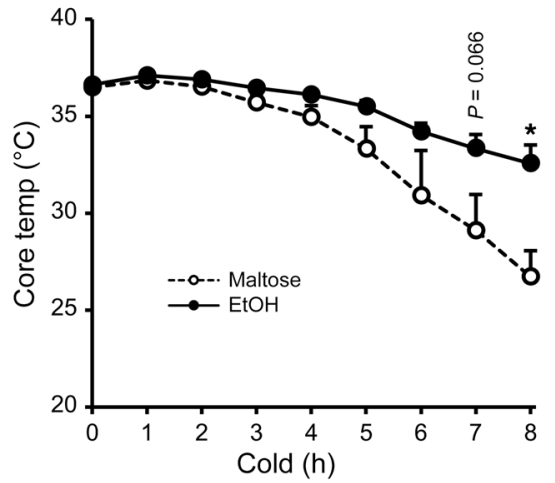

F
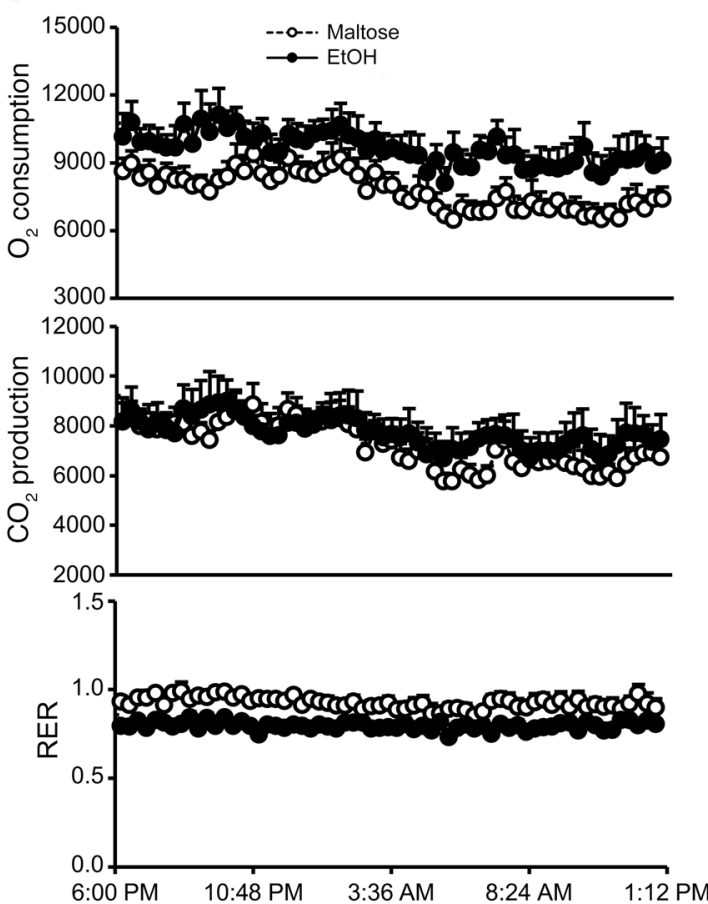

Figure 5. Alcohol consumption activates BAT. (A-D) C57BL/6 males were housed at $22^{\circ} \mathrm{C}$ or $30^{\circ} \mathrm{C}$ and fed alcohol or maltose diet for 13 weeks. (A) Representative BAT sections stained with H\&E or anti-Ucp1 antibody (>4 mice per group). Scale bar: $200 \mu \mathrm{m}$. (B) BAT extracts were immunoblotted with antibodies against Ucp1, Oxphos antibody cocktails, and p85 (loading control). (C) BAT OCR (normalized to BAT weight) ( $n=5$ mice per group). (D) Ucp1 mRNA levels in iWAT (normalized to $18 \mathrm{~S}$ levels) ( $n=5-6$ mice per group). (E, F) C57BL/6 males were housed at $22^{\circ} \mathrm{C}$ and fed alcohol $(n=5)$ or maltose diet $(n=5)$ for 13 weeks (E) Cold tolerance tests $\left(4^{\circ} \mathrm{C}, n=5\right.$ mice per group). (F) Energy expenditure was assessed using metabolic cages (normalized to lean mass) ( $n=6-7$ per group). Data are presented as mean \pm SEM. ${ }^{*} P<0.05$, 1-way ANOVA after Greenhouse-Geisser correction with subsequent Bonferroni's multiple comparisons.

To gain insights into alcohol-sensing circuits, we assessed neuronal activity using c-Fos as a marker. Central alcohol rapidly stimulated neuronal activities in the rostra raphe pallidus ( $\mathrm{rRPa})$ and dorsomedial hypothalamus (DMH), but not in the arcuate nucleus (ARC), medial preoptic area (MPO), and paraventricular hypothalamus (PVH) (Figure 6, H and I). DMH neurons monosynaptically activate iRPa premotor neurons, which project to sympathetic preganglionic neurons innervating BAT (26). Consistently, central alcohol significantly increased levels of BAT tyrosine hydroxylase (TH), a sympathetic nerve marker (Figure 6D).
To determine whether sympathetic innervation is required for alcohol stimulation of BAT, we performed unilateral denervation of BAT while the contralateral side was sham-treated as control. Mice were, after surgery, placed on an alcohol diet for 4 weeks. They were housed at thermoneutrality to reduce baseline sympathetic activity. Denervation did not affect BAT morphology and Ucp1 expression in maltose-fed mice (Figure 7A). Remarkably, alcohol intake drastically increased expression of Ucp1 and TH in sham-treated but not denervated BAT (Figure 7, A-C). Denervation also blocked the ability of alcohol to increase the levels of HSL 

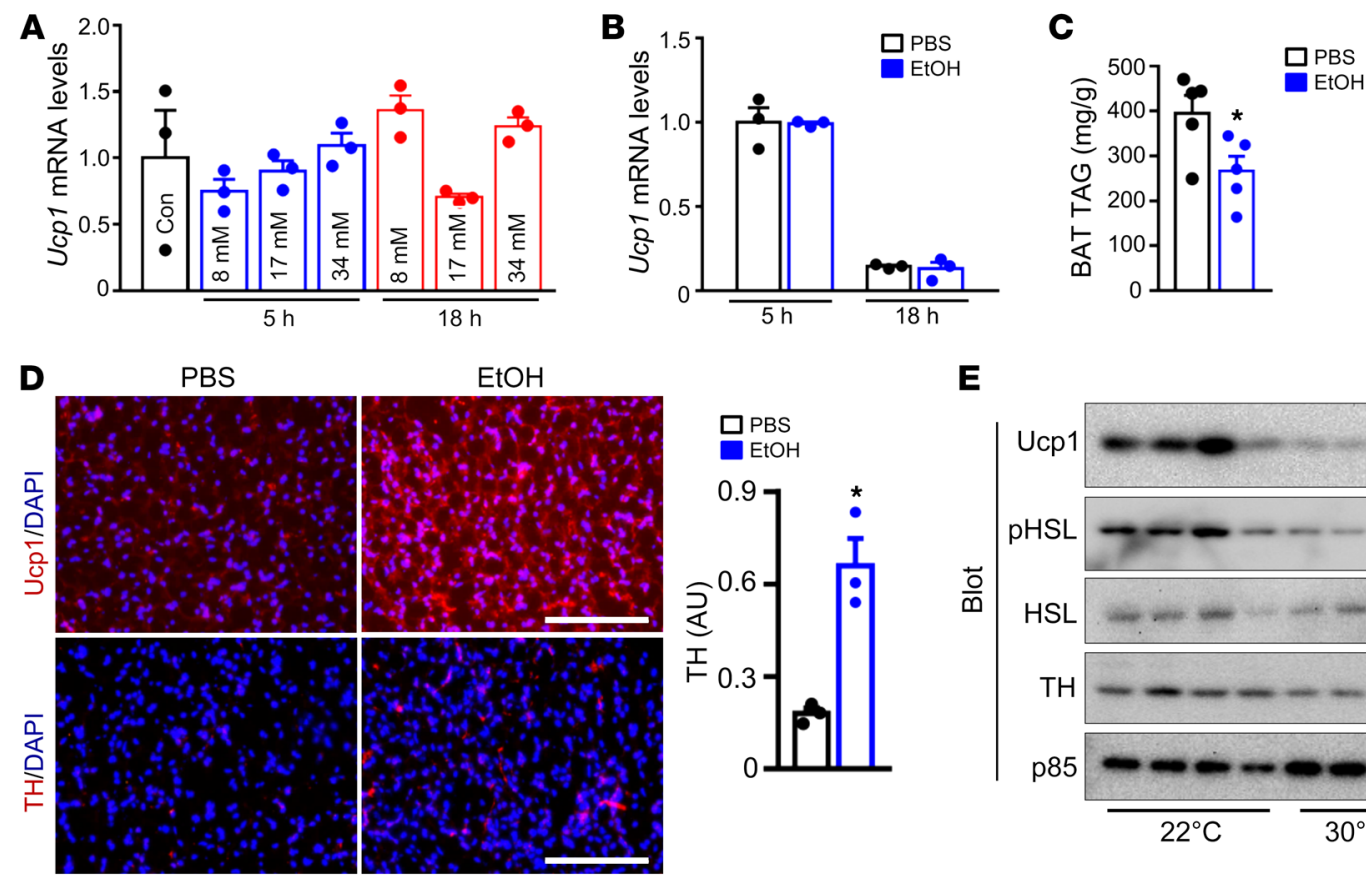

E
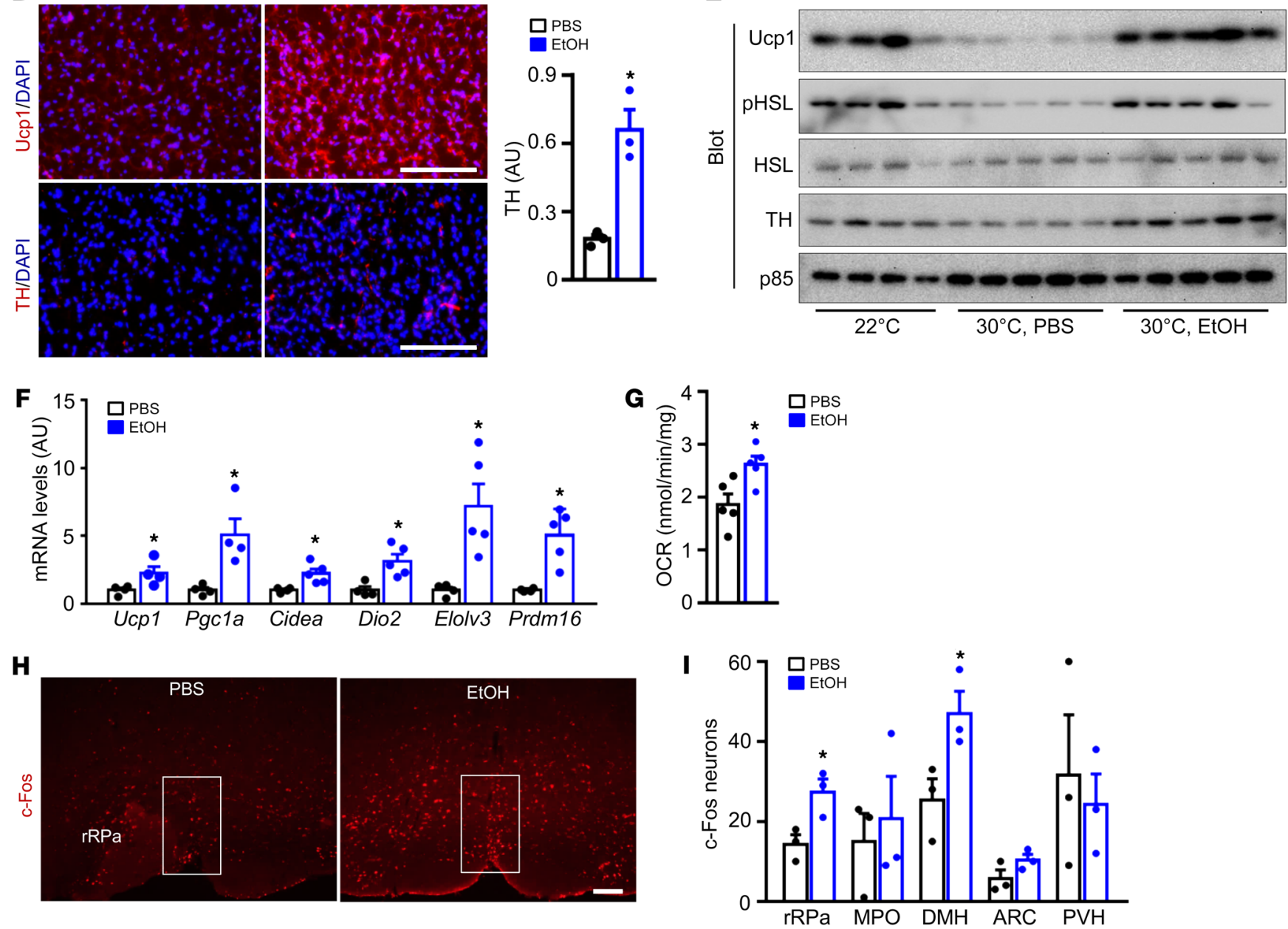

Figure 6. Brain alcohol-sensing activates BAT. (A-B) Differentiated (A) and primary (B) brown adipocytes were treated with ethanol for 5-18 hours. Ucp1 mRNA levels were measured by qPCR and normalized to $18 \mathrm{~S}$ levels ( $n=3$ per group). (C-C) C57BL/ 6 male mice were housed at $30^{\circ} \mathrm{C}$ and centrally administrated with alcohol for 3 weeks. (C) BAT TAC levels (normalized to BAT weight; $n=5$ per group). (D) Representative BAT sections. TH areas were normalized to the total areas ( $n=3$ mice per group). (E) BAT extracts were immunoblotted with the indicated antibodies. (F) BAT gene expression (normalized to 185 levels; $n=4-5$ per group). (C) BAT OCR (normalized to BAT weight, $n=5$ mice per group). (H, I) C57BL/6 males were centrally injected with alcohol, and brains were harvested 2 hours later. Brain sections were stained with anti-c-Fos antibody, and c-Fos ${ }^{+}$cells were quantified in different hypothalamic areas. PBS, $n=3$; EtOH, $n=3$. Scale bars: $200 \mu \mathrm{m}$. Data are presented as mean \pm SEM. ${ }^{*} P<0.05,2$-tailed unpaired Student's $t$ test.

phosphorylation, mitochondrial respiration complexes, OCR, and MMW adiponectin in BAT (Figure 7, D-F). Collectively, these results unravel an unrecognized brain alcohol-sensing/DMH/ $\mathrm{rRPa} /$ sympathetic nerve/BAT/liver axis (Figure 8).

\section{Discussion}

The pathogenic consequences of alcohol consumption have been extensively documented; counterintuitively, light drinking is also recognized to ameliorate type 2 diabetes, NAFLD, and cardiovas- cular diseases (13-22), but the underlying mechanism is unknown. Given that BAT and beige fat confer metabolic benefits by burning FFAs and glucose, we hypothesized that these tissues are the missing link between alcohol intake and health benefits. In the current study, we uncover a neural alcohol-sensing/sympathetic nerve/ BAT/liver pathway that counteracts alcoholic liver injury.

We provide multiple lines of evidence that establish a hypothalamus/sympathetic nerve/BAT axis. We found that chronic alcohol intake markedy increased Ucp1 expression in both BAT and beige 
A
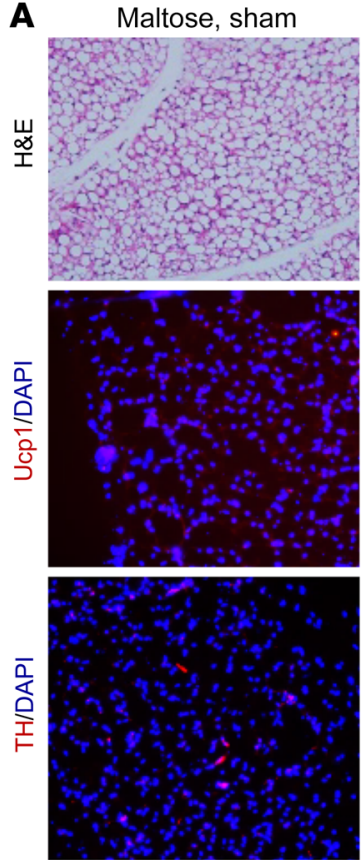

C

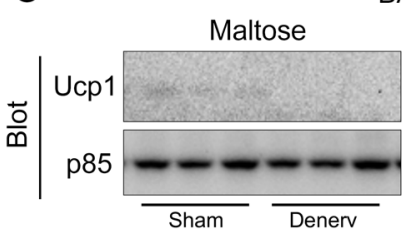

BAT

BAT

D
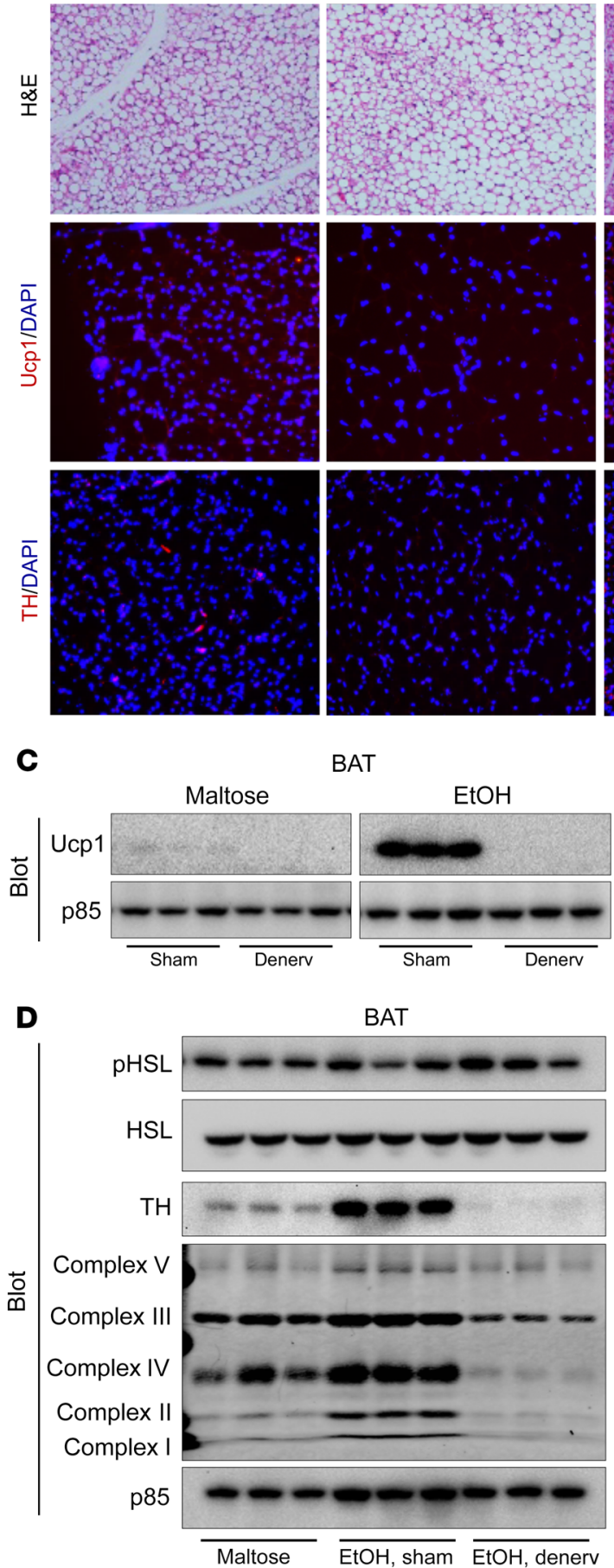

Maltose, denerv

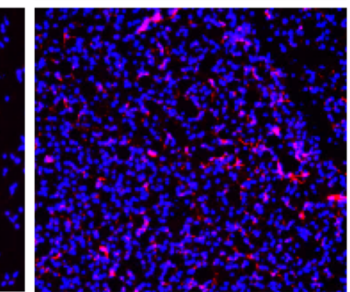

E

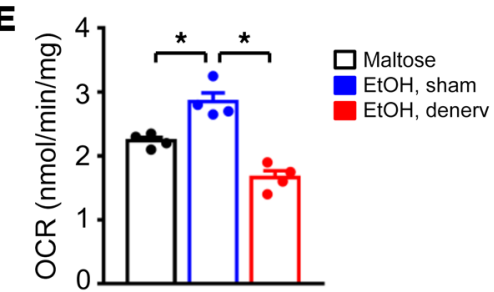

F

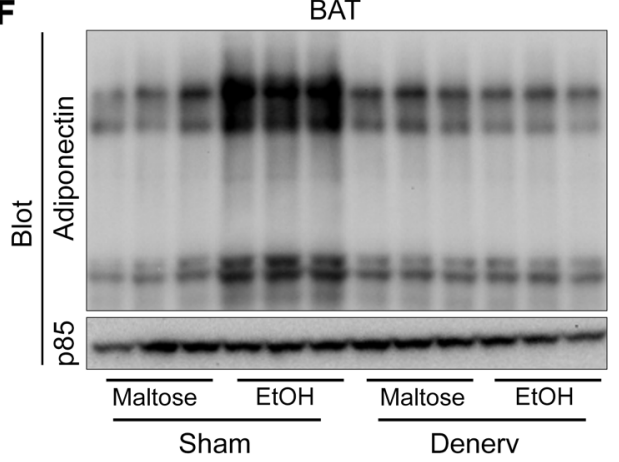

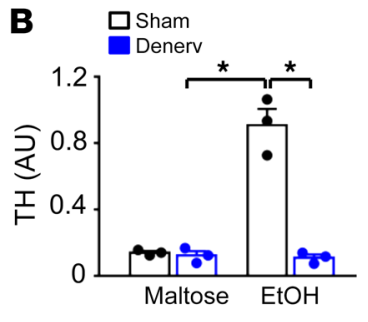
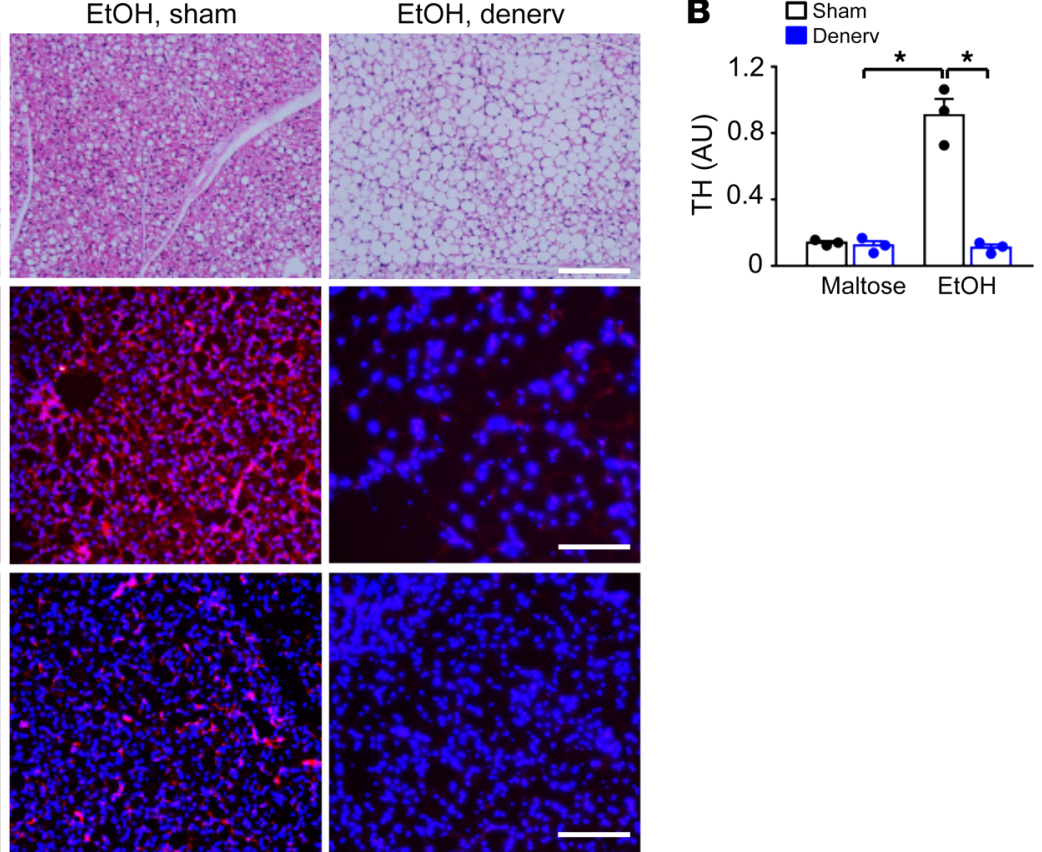


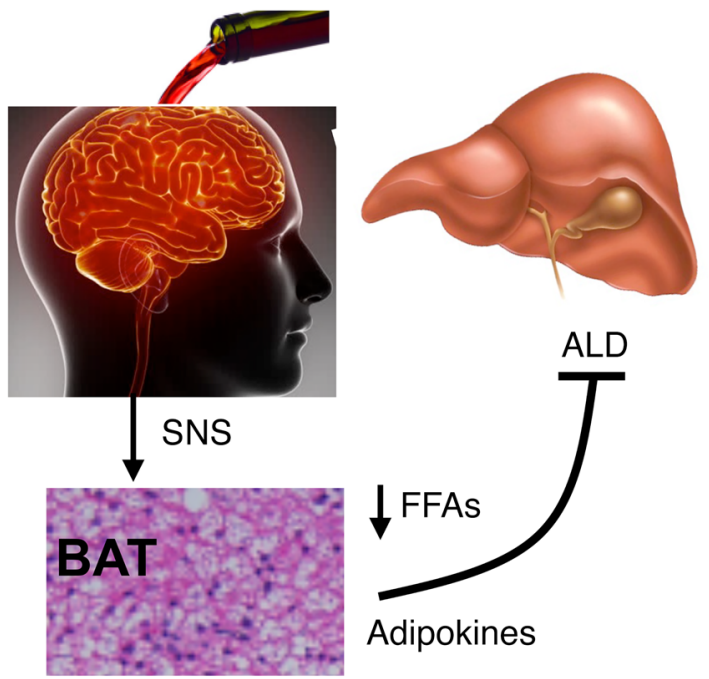

Figure 8. Protection against ALD by brain/SNS/BAT axis. Brain alcoholsensing activates BAT through increasing sympathetic inputs. BAT in turn protects against liver steatosis and injury by burning FFAs and secreting hepatoprotective adipokines.

BAT, indicating that brain alcohol sensing triggers activation of the sympathetic nerve/BAT axis. Furthermore, central alcohol directly stimulated neural activities in the DMH and rRPa, key nodes of the hypothalamic circuitry controlling the sympathetic nerve/BAT axis (26). Collectively, these results firmly establish the brain alcohol-sensing/sympathetic nerve/BAT axis (Figure 8). Notably, alcohol consumption increases brown adipocyte-like cells in heavy drinkers (46), suggesting that this axis has been conserved in humans.

We found that Ucp1 deficiency profoundly increases the severity of alcoholic liver steatosis, injury, and fibrosis in both male and female mice. The liver was normal in $U c \mathrm{p1}^{-1-}$ mice on a normal chow or maltose diet. In response to acute binge alcohol intake or chronic alcohol consumption, $U c \mathrm{P1}^{---}$mice developed substantially more severe liver steatosis compared with $\mathrm{Ucp}^{+1^{+/}}$mice. Conversely, activation of BAT and beige fat by cold exposure markedly suppressed alcoholic liver steatosis and oxidative stress. Notably, liver steatosis occurs in more than $90 \%$ of heavy alcohol drinkers and promotes liver injury and inflammation $(11,17,47)$. BAT and beige fat oxidize FFAs to fuel Ucp1-mediated thermogenesis, thereby decreasing circulating FFAs and consequently blocking lipid trafficking into the liver. In further support of this notion, $\beta_{3}$ adrenergic agonist treatment, which stimulates adipose lipolysis and release of FFAs, increased liver steatosis to a markedly higher degree in $\mathrm{UcP1}^{-/-}$relative to $\mathrm{Ucp}^{+/-}$mice. BAT was reported to support clearance of circulating FFAs in humans (48), suggesting that BAT protection against alcoholic liver steatosis has been conserved in humans. Remarkably, BAT or BAT-conditioned medium directly inhibited primary hepatocyte death ex vivo. Depletion of adiponectin or IL-6 attenuated the ability of BAT-conditioned medium to block hepatocyte death. These exciting findings demonstrate that BAT protects against ALD by both secreting hepatoprotective adipokines and burning FFAs (Figure 8). Considering that BAT and beige fat are able to ameliorate human NAFLD and metabolic disease $(24,49$,
50), we posit that the brain alcohol-sensing/sympathetic nerve/ BAT/beige fat axis likely confers a broad spectrum of health benefits beyond protection against ALD.

In conclusion, we unravel a brain alcohol-sensing/sympathetic nerve/BAT/liver axis that counteracts alcoholic liver steatosis, injury, inflammation, and fibrosis. This axis may serve as a potential therapeutic target for ALD.

\section{Methods}

Animals and ethanol feeding. Ucp1-knockout mice (C57BL/6 background) were from Jackson Laboratory. Male and female mice (7 to 8 weeks) were acclimated to the Lieber-DeCarli liquid diet (Bio-Serv, F1259SP) with alcohol concentrations increasing gradually, by $1 \%$ daily, to the final concentrations of $5 \%$ over 5 consecutive days (51). The mice were then fed a Lieber-DeCarli diet supplemented with $5 \%$ (vol/vol) ethanol (200\% proof) for an additional 7 to 13 weeks. As a control, mice were fed a Lieber-DeCarli liquid diet supplemented with isocaloric maltose $(71 \mathrm{~g} / \mathrm{l})$. For binge ethanol drinking, mice were acclimated to the Lieber-DeCarli ethanol diet for the first 5 days, followed by 10 days of feeding of the Lieber-DeCarli ethanol (5\%) diet. Afterwards, mice were treated with binge ethanol intake (5 g/ $\mathrm{kg}$ body weight, via gavage). Mice that displayed fatal hypothermia (rectal temperature below $22^{\circ} \mathrm{C}$ ), inability to move, and other severe symptoms of sickness were euthanized and considered as dead. In a separate cohort, mice were chronically fed an alcohol diet superimposed with binge drinking $(2.5 \mathrm{~g} / \mathrm{kg}$ body weight, twice a week). To avoid binge drinking-induced hypothermia, mice were placed at $30^{\circ} \mathrm{C}$ for the first 12 hours after binge drinking and then returned to $22^{\circ} \mathrm{C}$. C57BL/6 males (8 weeks) were housed for 10 days in an environmental chamber (RIS33SD, Innovative Solutions) at $8^{\circ} \mathrm{C}$ and treated with a single bout of binge alcohol ( $5 \mathrm{~g} / \mathrm{kg}$ body weight, gavage). The mice were treated twice ( 12 hours and 1 hour before binge alcohol intake) with anti-adiponectin ( $5 \mu \mathrm{g} /$ mouse) or PI (control) and euthanized at 9 hours after alcohol treatment.

Thermoneutrality, cold tolerance tests, and blood tests. Mice were housed at thermoneutrality $\left(30^{\circ} \mathrm{C}\right)$ in a temperature-controlled environmental chamber. For cold-tolerance tests, single-housed mice were placed at $4^{\circ} \mathrm{C}$, and rectal temperature was monitored hourly. Blood samples were collected from tail veins. Plasma ALT levels were measured using an ALT reagent set (Pointe Scientific Inc.).

Unilateral denervation. C57BL/6 male mice were anesthetized with isoflurane and shaved of hair on the nape regions. A lateral incision was made below shoulder blades, and intrascapular fat pads were retracted cranially over the head to expose 5 branches of intercostal sympathetic nerves subserving the left- and right-side BAT pads. The left branch was severed between roots and BAT, while the right branch was treated with a sham operation. The intrascapular fat pad was then returned to its original position and secured in place to the surrounding thoracic tissue and muscle using absorbable 6.0 Vicryl sutures. Mice were allowed 7 days of recovery prior to further experimental procedures.

Central alcohol administration. Mice were anesthetized with isoflurane and mounted on an Ultra Precise Small Animal Stereotaxic Alignment System (model 963, David Kopf Instruments). The skull was exposed, and a small opening was made using a bone drill at the following coordinates: $-0.34 \mathrm{~mm}(\mathrm{a}-\mathrm{p}), 1.0 \mathrm{~mm}(\mathrm{~m}-\mathrm{l})$, and $-2.4 \mathrm{~mm}(\mathrm{~d}-\mathrm{v})$. Intracerebroventricular cannula (C315GS-5/SPC, C315IS-5/SPC, Plastics One) were fixed to the skull using superglue and Teets denture 
material (Co-oral-ite Dental). Mice were allowed 7 days of recovery prior to intracerebroventricular injection of alcohol ( $5 \mu \mathrm{l}$ of $40 \%$ ethanol).

Metabolic cages and OCR. Energy expenditure was measured using indirect calorimetry (Windows Oxymax Equal Flow System, Columbus Instruments). Lean body mass was measured using a dual-energy $\mathrm{x}$-ray absorptiometry pDEXA (Norland Stratec). $\mathrm{O}_{2}$ consumption and $\mathrm{CO}_{2}$ production were normalized to lean mass. BAT was isolated and cut into small pieces $(1 \mathrm{mg} /$ piece). BAT explants were incubated in DMEM, and OCR was measured using the Mitocell Respiration Analysis System (MT200A, Strathkelvin Instruments).

Sirius red staining and hydroxyproline assays. Liver paraffin sections were stained with $0.1 \%$ Sirius red (MilliporeSigma, 365548) and 0.1\% Fast Green (MilliporeSigma, F7252) (in saturated picric acid). Liver samples were homogenized in $6 \mathrm{~N} \mathrm{HCl}$, hydrolyzed at $100^{\circ} \mathrm{C}$ for 18 hours, and centrifuged at $9400 \mathrm{~g}$ for 5 minutes. Supernatant was dried in speed vacuum, dissolved in $\mathrm{H}_{2} \mathrm{O}$, and neutralized with $10 \mathrm{~N}$ $\mathrm{NaOH}$. Samples were incubated in a chloramine-T solution $(60 \mathrm{mM}$ chloramines-T [MilliporeSigma, 857319], $20 \mathrm{mM}$ citrate, $50 \mathrm{mM}$ acetate, $\mathrm{pH}$ 6.5) for 25 minutes at room temperature and then in Ehrlich's solution (MilliporeSigma, 038910) at $65^{\circ} \mathrm{C}$ for an additional $20 \mathrm{~min}$ utes. Hydroxyproline content was measured using a Beckman Coulter AD 340 Plate Reader $(570 \mathrm{~nm})$ and normalized to liver weight.

Cell culture, CM, and chemical treatment. Primary hepatocytes were isolated by liver perfusion with type II collagenase (Worthington Biochemical Corp.) and grown in William's medium E (MilliporeSigma, W4125) supplemented with $2 \%$ FBS. BAT or WAT explants were prepared at a size of approximately $1 \mathrm{mg} /$ piece, and hepatocyte death was assessed using TUNEL assays. BAT or WAT explants were grown in William's medium E, and supernatants (i.e., CM) were collected 24 hours later. $\mathrm{CM}$ was pretreated with indicated antibodies (2 $\mu \mathrm{l} / \mathrm{ml}$ ) or PI (control) for 2 hours at $4^{\circ} \mathrm{C}$ and immune complexes were removed using protein A-agarose beads. Primary hepatocytes were treated fat explants, CM, and PA $(200 \mu \mathrm{M})$, as indicated, and hepatocyte death was assessed 24 hours later, using TUNEL assays.

Primary brown adipocytes and stromal vascular fraction cells (SVFs) were isolated from pups at 7 to 8 weeks of age using collagenase D (1.5 U/ml, Roche 11088882001) and Dispase II (2.4 U/ $\mathrm{ml}$, Roche 4942078001) and grown in DMEM supplemented with $4 \%$ BSA. Brown preadipocyte lines and SVFs were maintained in DMEM supplemented with $10 \%$ FBS. Confluent preadipocytes and SVFs were grown for 2 days and then cultured for an additional 3 days in differentiation DMEM supplemented with 10\% FBS, $0.5 \mathrm{mM}$ 3-isobutyl-1-methylxanthine (IBMX; MilliporeSigma, I-5879), $5 \mu \mathrm{M}$ dexamethasone (Sigma, D-1756), $0.125 \mathrm{mM}$ indomethacin (Sigma, I-7378), $20 \mathrm{nM}$ insulin, and $2 \mathrm{nM}$ T3 (Sigma, T-2877). The culture medium was replaced with DMEM supplemented with $20 \mathrm{nM}$ insulin and $2 \mathrm{nM} \mathrm{T3}$, and cells fully differentiated into brown adipocytes 3 to 4 days later.

Immunostaining and TUNEL assays. Liver frozen sections were prepared using a Leica cryostat (Leica Biosystems Nussloch GmbH), fixed in $4 \%$ paraformaldehyde for 30 minutes, blocked for 3 hours with $5 \%$ normal goat serum (Life Technologies) supplemented with
1\% BSA, and incubated with the indicated antibodies overnight at $4^{\circ} \mathrm{C}$. To assess cell death, liver sections were stained with TUNEL reagents using an In Situ Cell Death Detection Kit (Roche Diagnostics, 11684817910). To assess lipid droplets, liver sections were stained with Nile red (50 $\mathrm{ng} / \mathrm{ml}$ in PBS) for 30 minutes.

Immunoblotting. Cells or tissues were homogenized in L-RIPA lysis buffer (50 mM Tris, pH 7.5, 1\% Nonidet P-40, $150 \mathrm{mM} \mathrm{NaCl}, 2$ mM EGTA, $1 \mathrm{mM} \mathrm{Na}_{3} \mathrm{VO}_{4}, 100 \mathrm{mM} \mathrm{NaF}, 10 \mathrm{mM} \mathrm{Na}{ }_{4} \mathrm{P}_{2} \mathrm{O}_{7}, 1 \mathrm{mM}$ benzamidine, $10 \mu \mathrm{g} \mathrm{ml}^{-1}$ aprotinin, $10 \mu \mathrm{g} \mathrm{ml}^{-1}$ leupeptin, $1 \mathrm{mM}$ phenylmethylsulfonyl fluoride). Proteins were separated by SDS-PAGE and immunoblotted with the indicated antibodies. For adiponectin immunoblotting, experiments were conducted under both nonreducing and nonheating conditions. Antibodies are listed in Supplemental Table 1.

Real-time quantitative PCR and ROS assays. Total RNAs were extracted using TRIzol reagents (Life Technologies). Relative mRNA abundance was measured using Radiant SYBR Green $2 \times$ Lo-ROX qPCR Kits (Alkali Scientific) and StepOnePlus RT-PCR Systems (Life Technologies Corp.). Primers are listed in Supplemental Table 2. Liver lysates were mixed with a dichlorofluorescein diacetate fluorescent (DCF) (MilliporeSigma, D6883) probe $(5 \mu \mathrm{M})$ for 1 hour at $37^{\circ} \mathrm{C}$. DCF fluorescence was measured using a BioTek Synergy 2 Multi-Mode Microplate Reader (485 $\mathrm{nm}$ excitation and $527 \mathrm{~nm}$ emission).

Statistics. Data are presented as mean \pm SEM. Difference was analyzed between 2 groups by 2-tailed Student's $t$ test and between multiple groups by 1-way ANOVA after Greenhouse-Geisser correction with subsequent Bonferroni's multiple comparisons. $P<0.05$ was considered statistically significant.

Study approval. Animal experiments were conducted following the protocols approved by the University of Michigan IACUC.

Data availability. All relevant data are available upon request.

\section{Author contributions}

HS and LJ conducted the experiments, HS and LR designed the experiments and wrote the paper, JDL and MBO provided reagents, and HS, LJ, JDL, MBO, and LR performed data analysis and edited the paper.

\section{Acknowledgments}

We thank Yan Liu, Lei Yin, and Jun Wu for assistance and discussion. This study was supported by grants R01 DK091591, R01 DK094014 and R21 AA025945 (to LR); R01 DK102456 (to JDL); and R01 DK47918 (to MBO) from the NIH. This work utilized the cores supported by the Michigan Diabetes Research and Training Center (NIH DK20572), the University of Michigan's Cancer Center (NIH CA46592), the University of Michigan Nathan Shock Center (NIH P30AG013283), and the University of Michigan Center for Gastrointestinal Research (NIH DK34933).

Address correspondence to: Liangyou Rui, Department of Molecular \& Integrative Physiology, University of Michigan Medical School, Ann Arbor, Michigan 48109, USA. Phone: 734.615.7544; E-mail: ruily@umich.edu.
1. Grant BF, et al. Epidemiology of DSM-5 alcohol use disorder: Results from the National Epidemiologic Survey on Alcohol and Related Conditions III. JAMA Psychiatry. 2015;72(8):757-766.
2. Rehm J, Mathers C, Popova S, Thavorncharoensap M, Teerawattananon Y, Patra J. Global burden of disease and injury and economic cost attributable to alcohol use and alcohol-use disor- ders. Lancet. 2009;373(9682):2223-2233.

3. Zhong W, et al. Chronic alcohol exposure stimulates adipose tissue lipolysis in mice: role of reverse triglyceride transport in the 
pathogenesis of alcoholic steatosis. Am J Pathol. 2012;180(3):998-1007.

4. Zhao C, et al. FGF21 mediates alcohol-induced adipose tissue lipolysis by activation of systemic release of catecholamine in mice. JLipid Res. 2015;56(8):1481-1491.

5. Rachakonda V, et al. Stratification of risk of death in severe acute alcoholic hepatitis using a panel of adipokines and cytokines. Alcohol Clin Exp Res. 2014;38(11):2712-2721.

6. Kang L, et al. Chronic ethanol and triglyceride turnover in white adipose tissue in rats: inhibition of the anti-lipolytic action of insulin after chronic ethanol contributes to increased triglyceride degradation. J Biol Chem. 2007;282(39):28465-28473.

7. Yki-Järvinen H, Koivisto VA, Ylikahri R, Taskinen MR. Acute effects of ethanol and acetate on glucose kinetics in normal subjects. Am J Physiol. 1988;254(2 Pt 1):E175-E180.

8. Dou X, et al. Rectification of impaired adipose tissue methylation status and lipolytic response contributes to hepatoprotective effect of betaine in a mouse model of alcoholic liver disease. $\mathrm{Br} \mathrm{J}$ Pharmacol. 2014;171(17):4073-4086.

9. Marmier S, et al. Novel role for carbohydrate responsive element binding protein in the control of ethanol metabolism and susceptibility to binge drinking. Hepatology. 2015;62(4):1086-1100.

10. Beier JI, McClain CJ. Mechanisms and cell signaling in alcoholic liver disease. Biol Chem. 2010;391(11):1249-1264.

11. Louvet A, Mathurin P. Alcoholic liver disease: mechanisms of injury and targeted treatment. Nat Rev Gastroenterol Hepatol. 2015;12(4):231-242.

12. Szabo G, Petrasek J. Gut-liver axis and sterile signals in the development of alcoholic liver disease. Alcohol Alcohol. 2017;52(4):414-424.

13. Mukamal KJ, et al. Roles of drinking pattern and type of alcohol consumed in coronary heart disease in men. N Engl JMed. 2003;348(2):109-118.

14. Flanagan DE, Moore VM, Godsland IF, Cockington RA, Robinson JS, Phillips DI. Alcohol consumption and insulin resistance in young adults. Eur J Clin Invest. 2000;30(4):297-301.

15. Koppes LL, Dekker JM, Hendriks HF, Bouter LM, Heine RJ. Moderate alcohol consumption lowers the risk of type 2 diabetes: a meta-analysis of prospective observational studies. Diabetes Care. 2005;28(3):719-725.

16. Hong J, Smith RR, Harvey AE, Núñez NP. Alcohol consumption promotes insulin sensitivity without affecting body fat levels. Int JObes (Lond). 2009;33(2):197-203.

17. Bataller R, Gao B. Liver fibrosis in alcoholic liver disease. Semin Liver Dis. 2015;35(2):146-156.

18. Holst C, Becker U, Jørgensen ME, Grønbæk M, Tolstrup JS. Alcohol drinking patterns and risk of diabetes: a cohort study of 70,551 men and women from the general Danish population. Diabetologia. 2017;60(10):1941-1950.

19. Dunn W, et al. Modest alcohol consumption is associated with decreased prevalence of steatohepatitis in patients with non-alcoholic fatty liver disease (NAFLD). J Hepatol. 2012;57(2):384-391.

20. Sookoian S, Castaño GO, Pirola CJ. Modest alcohol consumption decreases the risk of nonalcoholic fatty liver disease: a meta-analysis of 43 175 individuals. Gut. 2014;63(3):530-532.

21. Ajmera VH, Terrault NA, Harrison SA. Is moderate alcohol use in nonalcoholic fatty liver disease good or bad? A critical review. Hepatology. 2017;65(6):2090-2099.

22. Hagström H, et al. Low to moderate lifetime alcohol consumption is associated with less advanced stages of fibrosis in non-alcoholic fatty liver disease. Scand J Gastroenterol. 2017;52(2):159-165.

23. Rosen ED, Spiegelman BM. What we talk about when we talk about fat. Cell. 2014;156(1-2):20-44.

24. Rui L. Brown and beige adipose tissues in health and disease. Compr Physiol. 2017;7(4):1281-1306.

25. Kajimura S, Spiegelman BM, Seale P. Brown and beige fat: physiological roles beyond heat generation. Cell Metab. 2015;22(4):546-559.

26. Morrison SF. Central neural control of thermoregulation and brown adipose tissue. Auton Neurosci. 2016;196:14-24.

27. Cypess AM, et al. Identification and importance of brown adipose tissue in adult humans. $N$ Engl J Med. 2009;360(15):1509-1517.

28. van Marken Lichtenbelt WD, et al. Cold-activated brown adipose tissue in healthy men. $\mathrm{NEnglJ}$ Med. 2009;360(15):1500-1508.

29. Virtanen KA, et al. Functional brown adipose tissue in healthy adults. $\mathrm{N}$ Engl J Med. 2009;360(15):1518-1525.

30. Yoneshiro T, et al. Recruited brown adipose tissue as an antiobesity agent in humans. J Clin Invest. 2013;123(8):3404-3408.

31. Blondin DP, et al. Four-week cold acclimation in adult humans shifts uncoupling thermogenesis from skeletal muscles to brown adipose tissue. JPhysiol (Lond). 2017;595(6):2099-2113.

32. Leitner BP, et al. Mapping of human brown adipose tissue in lean and obese young men. Proc Natl Acad Sci U S A. 2017;114(32):8649-8654.

33. van der Lans AA, et al. Cold acclimation recruits human brown fat and increases nonshivering thermogenesis. JClin Invest. 2013;123(8):3395-3403.

34. Chondronikola $\mathrm{M}$, et al. Brown adipose tissue activation is linked to distinct systemic effects on lipid metabolism in humans. Cell Metab. 2016;23(6):1200-1206.

35. Lowell BB, et al. Development of obesity in transgenic mice after genetic ablation of brown adipose tissue. Nature. 1993;366(6457):740-742.

36. Diehl AM, Chute J. Underlying potential: cellular and molecular determinants of adult liver repair. J Clin Invest. 2013;123(5):1858-1860.

37. Friedman SL. Mechanisms of hepatic fibrogenesis. Gastroenterology. 2008;134(6):1655-1669.

38. Richardson MM, et al. Progressive fibrosis in nonalcoholic steatohepatitis: association with altered regeneration and a ductular reaction. Gastroenterology. 2007;133(1):80-90.

39. Bertola A, Park O, Gao B. Chronic plus binge ethanol feeding synergistically induces neutrophil infiltration and liver injury in mice: a critical role for E-selectin. Hepatology. 2013;58(5):1814-1823.

40. Kalant H, Lê AD. Effects of ethanol on thermoregulation. Pharmacol Ther. 1983;23(3):313-364.

41. Steiner JL, Lang CH. Alcohol, adipose tissue and lipid dysregulation. Biomolecules. 2017;7(1):E16.

42. Xu A, Wang Y, Keshaw H, Xu LY, Lam KS, Cooper GJ. The fat-derived hormone adiponectin alleviates alcoholic and nonalcoholic fatty liver diseases in mice. JClin Invest. 2003;112(1):91-100.

43. Feldmann HM, Golozoubova V, Cannon B, Nedergaard J. UCP1 ablation induces obesity and abolishes diet-induced thermogenesis in mice exempt from thermal stress by living at thermoneutrality. Cell Metab. 2009;9(2):203-209.

44. Wang B, et al. Moderate alcohol intake induces thermogenic brown/beige adipocyte formation via elevating retinoic acid signaling. FASEB J. 2017;31(10):4612-4622.

45. Blaner WS, et al. Chronic alcohol consumption decreases brown adipose tissue mass and disrupts thermoregulation: a possible role for altered retinoid signaling. Sci Rep. 2017;7:43474.

46. Huttunen P, Kortelainen ML. Long-term alcohol consumption and brown adipose tissue in man. Eur J Appl Physiol Occup Physiol. 1990;60(6):418-424.

47. Mahli A, Hellerbrand C. Alcohol and obesity: a dangerous association for fatty liver disease. Dig Dis. 2016;34(Suppl 1):32-39.

48. Blondin DP, et al. Dietary fatty acid metabolism of brown adipose tissue in cold-acclimated men. Nat Commun. 2017;8:14146.

49. Yilmaz Y, et al. Association between the presence of brown adipose tissue and non-alcoholic fatty liver disease in adult humans. Aliment Pharmacol Ther. 2011;34(3):318-323.

50. Ozguven S, Ones T, Yilmaz Y, Turoglu HT, Imeryuz $\mathrm{N}$. The role of active brown adipose tissue in human metabolism. Eur J Nucl Med Mol Imaging. 2016;43(2):355-361.

51. Bertola A, Mathews S, Ki SH, Wang H, Gao B. Mouse model of chronic and binge ethanol feeding (the NIAAA model). Nat Protoc. 2013;8(3):627-637. 